\title{
An analytic solution for the equal-mass banana graph
}

\author{
Johannes Broedel, ${ }^{a}$ Claude Duhr, ${ }^{b, c}$ Falko Dulat, ${ }^{d}$ Robin Marzucca, ${ }^{c}$ Brenda Penante ${ }^{b}$ \\ and Lorenzo Tancredi ${ }^{b}$ \\ ${ }^{a}$ Institut für Mathematik und Institut für Physik, Humboldt-Universität zu Berlin, \\ IRIS Adlershof, Zum Grossen Windkanal 6, 12489 Berlin, Germany \\ ${ }^{b}$ Theoretical Physics Department, CERN, \\ Geneva, Switzerland \\ ${ }^{c}$ Center for Cosmology, Particle Physics and Phenomenology (CP3), \\ Université Catholique de Louvain, \\ Chemin du Cyclotron 2, 1348 Ottignies-Louvain-la-Neuve, Belgium \\ ${ }^{d}$ SLAC National Accelerator Laboratory, Stanford University, \\ 2575 Sand Hill Rd, Menlo Park, CA 94025, U.S.A. \\ E-mail: jbroedel@physik.hu-berlin.de, claude.duhr@cern.ch, \\ dulatf@slac.stanford.edu, robin.marzucca@uclouvain.be, \\ b. penante@cern.ch, lorenzo.tancredi@cern.ch
}

ABSTRACT: We present fully analytic results for all master integrals for the three-loop banana graph with four equal and non-zero masses. The results are remarkably simple and all integrals are expressed as linear combinations of iterated integrals of modular forms of uniform weight for the same congruence subgroup as for the two-loop equal-mass sunrise graph. We also show how to write the results in terms of elliptic polylogarithms evaluated at rational points.

KeYwords: NLO Computations, QCD Phenomenology

ArXiv EPRINT: 1907.03787 


\section{Contents}

1 Introduction 1

2 The banana graph 3

2.1 Notations and conventions 3

2.2 The system of differential equations satisfied by the banana family 5

$\begin{array}{ll}2.3 & \text { The fundamental solution matrix }\end{array}$

3 The geometry associated to the two-loop sunrise graph $\quad 8$

3.1 Relating the equal-mass banana and sunrise graphs 8

$\begin{array}{lll}3.2 & \text { The elliptic curve associated to the sunrise graph } & 11\end{array}$

3.3 A class of iterated integrals of modular forms for $\Gamma_{1}(6) \quad 13$

$\begin{array}{ll}3.4 & \text { The sunrise integral and modular forms for } \Gamma_{1}(6) \\ \end{array}$

4 Analytic results for the equal-mass banana graph $\quad \mathbf{1 6}$

4.1 The equal-mass banana graph and modular forms for $\Gamma_{1}(6) \quad 16$

$\begin{array}{lll}4.2 & \text { Solution in terms of elliptic polylogarithms } & 19\end{array}$

5 Conclusion and outlook $\quad 23$

$\begin{array}{ll}\text { A Boundary condition for the banana graph } & 23\end{array}$

B Decomposing a matrix into a semi-simple and a unipotent part $\quad 25$

\section{Introduction}

Feynman integrals are the most important building blocks required to study scattering processes in perturbative quantum field theory. The physics program at the Large Hadron Collider has benefitted dramatically from the availability of theoretical predictions with high degree of accuracy. These predictions were made possible by tremendous advancements in the calculation of multi-loop scattering amplitudes in recent years. However, it has become clear that future efforts to further test our theoretical understanding of the nature of particle interactions at high energies will require an even higher level of precision. Computing scattering processes to even higher orders in perturbation theory will therefore require a deeper understanding of multi-loop Feynman integrals and a further refinement of the mathematical technology used to evaluate them.

Feynman integrals encode the complicated branch cut structure of scattering amplitudes, reflecting the structure of physical thresholds of scattering processes. Consequently, Feynman integrals need to be described in terms of classes of special functions that exhibit the required branch cuts. The classic examples are the logarithm and dilogarithm functions 
that encode the branch cut structure of simple one-loop amplitudes in four space-time dimensions. More complicated Feynman integrals require functions with a richer analytical structure in order to properly encode their branch cut structure. In this context, multiple polylogarithms [1-3] have proven an amazingly successful class of functions to describe many scattering processes, in particular in phenomenologically interesting cases where no massive particles circulate inside the loops.

However, it is well known that MPLs do not exhaust the space of functions to which Feynman integrals evaluate. It particular, it has been known for several decades that starting from two loops not all Feynman integrals evaluate to MPLs [4-16], though no complete analytic results were known. This situation has changed with the work of Bloch and Vanhove [17], who have shown that the simplest example of a Feynman integral that cannot be evaluated in terms of MPLs is in fact expressible through a generalisation of the dilogarithm to an elliptic curve. This result has sparked a lot of activity over the last few years, and by now we have complete analytic results for many Feynman integrals that involve functions of elliptic type [18-40]. In all cases these results involve new classes of transcendental functions, related either to elliptic generalisations of MPLs [17, 41-43] or iterated integrals of modular forms [35, 44-46]. Incidentally, these are also the same class of functions which describe string amplitudes at genus one [47-50].

It is also known that functions related to more complicated geometries show up [11, 14, 51-55]. The simplest example of such an integral is probably the three-loop banana graph with four massive propagators, whose associated geometry is a specific family of K3 surfaces [51]. While functions of elliptic type that arise in Feynman integral computations start to be well understood, we still lack a clear picture of the class of functions that arise from more complicated geometries. Hence, no complete analytic results are known for the banana graph in terms of a well-defined class of transcendental functions.

An exception to this case is the limit where all four masses in the banana graph are equal. In this case the K3 surface is elliptically fibered, and the base and the fiber are described by the same elliptic curve. This elliptic curve, in turn, is related to the elliptic curve of the sunrise integral [51]. The corresponding family of K3 surfaces and their associated Picard-Fuchs operator were studied in ref. [56], where it was shown that the solutions of this operator can be written in terms of the solutions of the Picard-Fuchs operator of the sunrise graph. In ref. [57] this property was used to express all master integrals for the equal-mass banana graph in terms of iterated integrals whose integration kernels involve products of complete elliptic integrals. However, a complete analytic solution of all master integrals for the equal-mass banana graph in terms of a well-defined and well-studied class of functions is currently still lacking.

In the remainder of this paper we close this gap and we present for the first time complete analytic results for all three master integrals of the equal-mass three-loop banana graph in $d=2$ dimensions. Our starting point is the differential equation of refs. [56, 57]. From there we show that, since the homogeneous solutions can be expressed in terms of the same modular forms that appear in the computation of the sunrise graph, the differential equation for the master integrals of the banana graph can be solved in terms of the same class of functions as for the sunrise graph. When expressed in this way, our results are 
characterised by a remarkable simplicity. Moreover, we observe that all master integrals can be written as linear combinations of pure functions of uniform weight, as defined in ref. [36].

The paper is organised as follows: in section 2 we review the banana graph, its differential equations and the results of refs. [51] and [57]. In section 3 we illustrate how to solve the differential equation of the sunrise graph in terms of iterated integrals of modular forms, and we introduce the relevant class of functions. In section 4 we present our main result, i.e., analytic results for all master integrals of the banana graph in $d=2$ dimensions in terms of iterated integrals of modular forms and in terms of elliptic polylogarithms. Finally, in section 5 we draw our conclusions. We include additional appendices where we discuss how to obtain the boundary conditions for the system of differential equations for the banana graph and where we present a method to decompose an invertible matrix into a product of a lower and an upper-triangular matrix.

\section{The banana graph}

\subsection{Notations and conventions}

The banana graph depicted in figure 1 constitutes one of the simplest families of a threeloop Feynman graph. Whenever at least either two propagator masses or the external invariant vanish, all members of the family can be expressed in terms of standard multiple polylogarithms (see e.g. ref. [58]). If all propagators are massive, new classes of functions are known to show up [51,57], related to a specific family of K3 surfaces. Not much is known in the most general case and in particular no analytic result is known for the banana family with distinct propagator masses.

Here we focus on a scenario of intermediate complexity, namely the case where all internal masses are chosen to be different from zero and equal. More precisely, let us consider the family of integrals defined by

$$
\begin{aligned}
& I_{a_{1}, \ldots, a_{9}}\left(p^{2}, m^{2} ; d\right)= \\
& \quad=\int \prod_{i=1}^{3} \mathfrak{D}^{d} \ell_{i} \frac{\left(\ell_{3}^{2}\right)^{a_{5}}\left(\ell_{1} \cdot p\right)^{a_{6}}\left(\ell_{2} \cdot p\right)^{a_{7}}\left(\ell_{3} \cdot p\right)^{a_{8}}\left(\ell_{1} \cdot \ell_{2}\right)^{a_{9}}}{\left[\ell_{1}^{2}-m^{2}\right]^{a_{1}}\left[\ell_{2}^{2}-m^{2}\right]^{a_{2}}\left[\left(\ell_{1}-\ell_{3}\right)^{2}-m^{2}\right]^{a_{3}}\left[\left(\ell_{2}-\ell_{3}-p\right)^{2}-m^{2}\right]^{a_{4}}},
\end{aligned}
$$

where the $a_{i} \geq 0$ are positive integers, and we have introduced the integration measure

$$
\int \mathfrak{D}^{d} \ell=\frac{1}{\Gamma\left(2-\frac{d}{2}\right)} \int \frac{d^{d} \ell}{i \pi^{d / 2}}
$$

Since all integrals depend on $p^{2}$ and $m^{2}$ only, it is convenient to express their non-trivial functional dependence in terms of the dimensionless ratio

$$
x=\frac{4 m^{2}}{p^{2}} .
$$

Furthermore, in what follows we will set $m=1$ for simplicity, since the dependence on $m$ of the different integrals can be recovered by dimensional analysis. The integrals may diverge in $d=4$ dimensions. We therefore work in dimensional regularisation where $d=d_{0}-2 \epsilon$ 


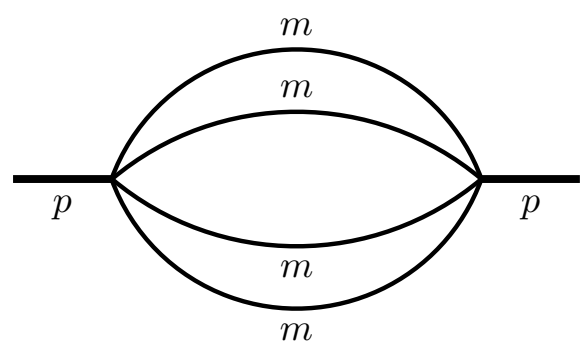

Figure 1. The three-loop banana graph.

with $d_{0}>0$ a positive integer. Accordingly, all integrals are interpreted as a Laurent series in the dimensional regulator $\epsilon$.

Let us now focus on the integrals in eq. (2.1). Using integration-by-parts identities [59, 60], we can express any member of this integral family in terms of four distinct master integrals. Moreover, we can use dimensional shift identities [61-66] to relate the coefficients of the Laurent expansion of these master integrals in $d=4-2 \epsilon$ to the corresponding ones in $d=2-2 \epsilon$ dimensions. Indeed, since all integrals are IR finite, by lowering the number of dimensions we improve their UV behaviour. This allows us to choose a finite basis of master integrals as follows

$$
\begin{aligned}
& \mathcal{I}_{1}(\epsilon ; x)=(1+2 \epsilon)(1+3 \epsilon) I_{1,1,1,1,0,0,0,0,0}\left(p^{2}, 1 ; 2-2 \epsilon\right), \\
& \mathcal{I}_{2}(\epsilon ; x)=(1+2 \epsilon) I_{2,1,1,1,0,0,0,0,0}\left(p^{2}, 1 ; 2-2 \epsilon\right), \\
& \mathcal{I}_{3}(\epsilon ; x)=I_{2,2,1,1,0,0,0,0,0}\left(p^{2}, 1 ; 2-2 \epsilon\right),
\end{aligned}
$$

where $x$ has been defined in eq. (2.3). The fourth master integral is the three-loop tadpole with squared propagators, which in our normalisation evaluates to

$$
\mathcal{I}_{0}(\epsilon ; x)=I_{2,2,2,0,0,0,0,0,0}\left(p^{2}, 1 ; 2-2 \epsilon\right)=1 .
$$

The main goal of this paper is to present analytic results for the three master integrals in eq. (2.4) in $d=2$ dimensions, i.e. for $\epsilon=0$. We stress that this is sufficient to obtain results in $d=4-2 \epsilon$ dimensions up to terms that are suppressed by powers of $\epsilon$, as one can easily verify from the relevant dimensional shift relations [61, 66]. As an example, the relation needed to express the master integral $\mathcal{I}_{1}(d ; x)$ in terms of the four masters in $d-2$ dimensions reads

$$
\begin{aligned}
\mathcal{I}_{1}(d ; x)= & c_{1}(d ; x) \mathcal{I}_{1}(d-2 ; x)+c_{2}(d ; x) \mathcal{I}_{2}(d-2 ; x)+c_{3}(d ; x) \mathcal{I}_{3}(d-2 ; x) \\
& +c_{0}(d ; x) \mathcal{I}_{0}(d-2 ; x),
\end{aligned}
$$

where the coefficients are

$$
\begin{aligned}
& c_{1}(d ; x)=\frac{1-20 x}{3 x}+\mathcal{O}(d-4), \\
& c_{2}(d ; x)=\frac{12-16 x(5+7 x)}{3 x^{2}}+\mathcal{O}(d-4), \\
& c_{3}(d ; x)=\frac{8(1-4 x)(1+2 x(5+2 x))}{3 x^{3}}+\mathcal{O}(d-4), \\
& c_{4}(d ; x)=-2+\mathcal{O}(d-4) .
\end{aligned}
$$


Inspecting eq. (2.6), we see that expanding the left hand side around $d=4$ corresponds to expanding the integrals on the right hand side in two dimensions. Since all coefficients in eq. (2.7) are finite in this limit, this implies that the finite pieces of the master integrals expanded close to $d=2$ are sufficient to obtain the finite terms of the corresponding integrals in $d=4$. Moreover, since the only divergent integral on the right hand side is $\mathcal{I}_{0}(d ; x)$, it is clear that the poles of the banana integrals close to $d=4$ arise exclusively from the tadpole integral.

\subsection{The system of differential equations satisfied by the banana family}

It is well known that master integrals satisfy differential equations in the external kinematic variables [67-71]. For the three non-trivial master integrals of the banana graph defined in the previous subsection, this system of differential equations can be written as [57]

$$
\partial_{x}\left(\begin{array}{l}
\mathcal{I}_{1}(\epsilon ; x) \\
\mathcal{I}_{2}(\epsilon ; x) \\
\mathcal{I}_{3}(\epsilon ; x)
\end{array}\right)=[B(x)+\epsilon D(x)]\left(\begin{array}{l}
\mathcal{I}_{1}(\epsilon ; x) \\
\mathcal{I}_{2}(\epsilon ; x) \\
\mathcal{I}_{3}(\epsilon ; x)
\end{array}\right)+\left(\begin{array}{c}
0 \\
0 \\
-\frac{1}{2(4 x-1)}
\end{array}\right)
$$

where the matrices $B(x)$ and $D(x)$ are given by

$$
\begin{aligned}
& B(x)=\left(\begin{array}{ccc}
\frac{1}{x} & \frac{4}{x} & 0 \\
\frac{1}{4(1-x)} & \frac{1}{x}+\frac{2}{1-x} & \frac{3}{x}+\frac{3}{1-x} \\
-\frac{1}{8(1-x)}+\frac{1}{8(1-4 x)} & -\frac{1}{1-x}+\frac{3}{2(1-4 x)} & \frac{1}{x}+\frac{6}{1-4 x}-\frac{3}{2(1-x)}
\end{array}\right), \\
& D(x)=\left(\begin{array}{ccc}
\frac{3}{x} & \frac{12}{x} & 0 \\
\frac{1}{1-x} & \frac{2}{x}+\frac{6}{1-x} & \frac{6}{x}+\frac{6}{1-x} \\
-\frac{1}{2(1-x)}+\frac{1}{2(1-4 x)} & -\frac{3}{1-x}+\frac{9}{2(1-4 x)} & \frac{1}{x}+\frac{12}{1-4 x}-\frac{3}{1-x}
\end{array}\right) .
\end{aligned}
$$

The inhomogeneity arises from the tadpole master integral in eq. (2.5), which does not depend on $x$ and therefore decouples entirely from the system of differential equations. Since in this paper we are only concerned with the value of the integrals in $d=2$ dimensions, we can let $\epsilon=0$ in eq. (2.8), which removes the dependence on the matrix $D(x)$. From now on we therefore focus on this simpler system. Introducing the shorthand $\mathcal{I}_{i}(x) \equiv \mathcal{I}_{i}(0 ; x)$ for the master integrals evaluated at $\epsilon=0$, the system reads

$$
\partial_{x}\left(\begin{array}{l}
\mathcal{I}_{1}(x) \\
\mathcal{I}_{2}(x) \\
\mathcal{I}_{3}(x)
\end{array}\right)=B(x)\left(\begin{array}{l}
\mathcal{I}_{1}(x) \\
\mathcal{I}_{2}(x) \\
\mathcal{I}_{3}(x)
\end{array}\right)+\left(\begin{array}{c}
0 \\
0 \\
-\frac{1}{2(4 x-1)}
\end{array}\right)
$$

Let us sketch how to solve this system using the method of variation of constants. Assume that we can find a fundamental solution matrix to eq. (2.11), i.e., a $3 \times 3$ matrix $\mathcal{W}(x)$ satisfying the homogeneous equation associated to eq. (2.11),

$$
\partial_{x} \mathcal{W}(x)=B(x) \mathcal{W}(x) .
$$

We then see that the vector $\left(M_{1}(x), M_{2}(x), M_{3}(x)\right)^{T}$ defined as

$$
\left(\begin{array}{l}
\mathcal{I}_{1}(x) \\
\mathcal{I}_{2}(x) \\
\mathcal{I}_{3}(x)
\end{array}\right)=\mathcal{W}(x)\left(\begin{array}{l}
M_{1}(x) \\
M_{2}(x) \\
M_{3}(x)
\end{array}\right)
$$


satisfies the inhomogeneous system of differential equations

$$
\partial_{x}\left(\begin{array}{l}
M_{1}(x) \\
M_{2}(x) \\
M_{3}(x)
\end{array}\right)=\mathcal{W}^{-1}(x)\left(\begin{array}{c}
0 \\
0 \\
-\frac{1}{2(4 x-1)}
\end{array}\right),
$$

which can now easily be solved by quadrature. We note that $\mathcal{W}(x)$ is always invertible for generic values of $x$ because its columns span the three-dimensional solution space of the homogeneous system in eq. (2.12) and are therefore linearly independent.

Solving the differential equation involves then two steps:

1. Finding a fundamental solution matrix $\mathcal{W}(x)$ satisfying the homogeneous differential equation in eq. (2.12).

2. Solving eq. (2.14) by quadrature. This involves in particular computing integrals over (products of) the entries of the fundamental solution matrix.

In the remainder of this section we review how to construct the fundamental solution matrix $\mathcal{W}(x)$ in the case of the equal-mass banana graph. The entries of $\mathcal{W}(x)$ are in general transcendental functions, so that the second step will involve the computation of integrals over transcendental functions. The main goal of this paper is to show how these integrals can be systematically performed in terms of iterated integrals over known objects.

\subsection{The fundamental solution matrix}

In general, it can be very complicated to find the fundamental solution matrix of a system of differential equations. In the case of Feynman integrals, the fundamental solution matrix can be obtained by studying the maximal cut of the integrals $[23,72,73]$. For the equal-mass banana graph, there is an alternative way to solve the homogeneous differential equation in eq. (2.12) [51, 57], which we review in the remainder of this section.

It will be convenient to introduce the following parametrisation of the fundamental solution matrix,

$$
\mathcal{W}(x)=\left(\begin{array}{lll}
H_{1}(x) & J_{1}(x) & I_{1}(x) \\
H_{2}(x) & J_{2}(x) & I_{2}(x) \\
H_{3}(x) & J_{3}(x) & I_{3}(x)
\end{array}\right) .
$$

Next, we convert the linear first-order system of differential equations in eq. (2.12) into a third-order differential equation for the first line of $\mathcal{W}(x)$,

$$
\mathcal{L}_{x}^{(3)} H_{1}(x)=\mathcal{L}_{x}^{(3)} J_{1}(x)=\mathcal{L}_{x}^{(3)} I_{1}(x)=0
$$

where $\mathcal{L}_{x}^{(3)}$ is the third-order linear differential operator [51, 57]

$$
\mathcal{L}_{x}^{(3)}=\partial_{x}^{3}+\frac{3(8 x-5)}{2(x-1)(4 x-1)} \partial_{x}^{2}+\frac{4 x^{2}-2 x+1}{(x-1)(4 x-1) x^{2}} \partial_{x}+\frac{1}{x^{3}(4 x-1)} .
$$


This can be achieved in a standard way by using the system of differential equations to re-express $H_{2}(x)$ and $H_{3}(x)$ in terms of $H_{1}(x)$ and its derivatives, namely

$$
\begin{aligned}
& H_{2}(x)=\frac{1}{4}\left(x \partial_{x}-1\right) H_{1}(x) \\
& H_{3}(x)=\frac{1}{12}\left(x^{2}(1-x) \partial_{x}^{2}-x(1+x) \partial_{x}+1\right) H_{1}(x)
\end{aligned}
$$

yielding in this way a third-order differential equation satisfied by $H_{1}(x)$. If a solution for $H_{1}(x)$ can be found by solving this higher-order equation, the corresponding solutions for $H_{2}(x)$ and $H_{3}(x)$ can be recovered by differentiating $H_{1}(x)$ according to eq. (2.18). The same reasoning can of course be applied to the other two columns of $\mathcal{W}(x)$, i.e. to the solutions $J_{i}(x)$ and $I_{i}(x)$.

In general, solving a third-order differential equation is a formidable task, and no general algorithm is known for finding the kernel of a generic third-order linear differential operator. It turns out, however, that the operator $\mathcal{L}_{x}^{(3)}$ is very special, and its solution can be expressed in terms of the solutions to the following second-order differential operator,

$$
\mathcal{L}_{x}^{(2)}=\partial_{x}^{2}+\frac{8 x-5}{2(x-1)(4 x-1)} \partial_{x}-\frac{2 x-1}{4 x^{2}(x-1)(4 x-1)} .
$$

Specifically, $\mathcal{L}_{x}^{(3)}$ is the symmetric square of the operator $\mathcal{L}_{x}^{(2)}$ [74], meaning that the three independent solutions of $\mathcal{L}_{x}^{(3)}$ are the products of the two independent solutions of $\mathcal{L}_{x}^{(2)}$. The solutions of $\mathcal{L}_{x}^{(2)}$, in turn, can be expressed in terms of complete elliptic integrals of the first kind. With this insight, one finds that the three independent homogeneous solutions can be suitably written as $[57,74]$

$$
\begin{aligned}
H_{1}(x) & =\sqrt{\lambda_{+}(x) \lambda_{-}(x)} \mathrm{K}\left(\lambda_{+}(x)\right) \mathrm{K}\left(\lambda_{-}(x)\right), \\
J_{1}(x) & =\sqrt{\lambda_{+}(x) \lambda_{-}(x)} \mathrm{K}\left(\lambda_{+}(x)\right) \mathrm{K}\left(1-\lambda_{-}(x)\right), \\
I_{1}(x) & =\sqrt{\lambda_{+}(x) \lambda_{-}(x)} \mathrm{K}\left(1-\lambda_{+}(x)\right) \mathrm{K}\left(1-\lambda_{-}(x)\right),
\end{aligned}
$$

where we defined

$$
\lambda_{ \pm}(x)=\frac{4 x}{2 x+(1-2 x) \sqrt{\frac{x-1}{x}} \pm \sqrt{\frac{4 x-1}{x}}},
$$

and $\mathrm{K}$ denotes the complete elliptic integral of the first kind

$$
\mathrm{K}(\lambda)=\int_{0}^{1} \frac{d t}{\sqrt{\left(1-t^{2}\right)\left(1-\lambda t^{2}\right)}} .
$$

By inspecting eq. (2.20), one might wonder why we have used four apparently independent building blocks to construct the solutions, i.e. $\mathrm{K}\left(\lambda_{+}(x)\right), \mathrm{K}\left(\lambda_{-}(x)\right), \mathrm{K}\left(1-\lambda_{+}(x)\right)$, and $\mathrm{K}\left(1-\lambda_{-}(x)\right)$, when we stated explicitly that all three solutions can be written as products of only two independent functions. Indeed, the four functions above are not independent and the explicit relations among them are non-trivial as they require to cross the branch cut of $\mathrm{K}(x)$ and therefore depend on the prescription we adopt to do so. Instead, working with an over-complete number of functions has the advantage of allowing us to choose a 
compact representation for the solutions, which have the correct analytic properties. For an explicit solution in terms of two functions only, see eq. (3.13) in the next section.

We have thus obtained the components of the first row of the fundamental solution matrix in eq. (2.15). The other rows can be obtained from eq. (2.18): they involve derivatives of complete elliptic integrals of the first kind that are expressible in terms of complete elliptic integrals of the second kind,

$$
\mathrm{E}(\lambda)=\int_{0}^{1} d t \sqrt{\frac{1-\lambda t^{2}}{1-t^{2}}} .
$$

The complete set of results for the fundamental solution matrix can be found for example in ref. [57].

The previous discussion makes it clear that, upon inserting the solution for $\mathcal{W}(x)$ into eq. (2.14), the $M_{i}(x)$ will naturally be expressed as integrals over products of complete elliptic integrals of the first and second kind. This program was carried out in ref. [57]. It is a priori not obvious if/how these new classes of iterated integrals can be expressed in terms of other classes of special functions that have appeared in Feynman integral computations and/or pure mathematics. The main goal of this paper is to show that it is indeed possible to express all master integrals for the banana family in terms of a known set of special functions: the class of functions that naturally appear in the solution of the two-loop sunrise integral family with three equal masses. The connection between the two families of integrals will be explored in more detail in the next section before we return to our original problem.

\section{The geometry associated to the two-loop sunrise graph}

\subsection{Relating the equal-mass banana and sunrise graphs}

The purpose of this subsection is to set the stage for the mathematical objects that will appear in the analytic result for the master integrals of the equal-mass banana graph in $d=2$ dimensions presented in section 4 . As anticipated at the end of the previous section, the relevant functions will essentially be identical to those appearing in the computation of the equal-mass two-loop sunrise family

$$
S_{a_{1}, \ldots, a_{5}}\left(p^{2}, m^{2} ; d\right)=\int \mathfrak{D}^{d} \ell_{1} \mathfrak{D}^{d} \ell_{2} \frac{\left(\ell_{1} \cdot p\right)^{a_{4}}\left(\ell_{2} \cdot p\right)^{a_{5}}}{\left[\ell_{1}^{2}-m^{2}\right]^{a_{1}}\left[\ell_{2}^{2}-m^{2}\right]^{a_{2}}\left[\left(\ell_{1}-\ell_{2}-p\right)^{2}-m^{2}\right]^{a_{3}}},
$$

where the integration measure was defined in eq. (2.2).

It has been known for a long time $[4,75]$ that in the case where all three propagators are massive, the sunrise integral cannot be expressed in terms of polylogarithmic functions, but instead requires the introduction of functions related to elliptic integrals.

By now we know several analytic representations for the sunrise family, all of which require the introduction of new classes of functions which generalise multiple polylogarithms and elliptic integrals. In the remainder of this section we review the class of functions relevant to the sunrise graph. As we will see in section 4 below, some of these classes of functions also appear in the banana graph. 
We start with some general facts about the sunrise family. Since all of these results are in principle well known (cf., e.g., refs. [7, 22]), and all the technical steps are very similar to the case of the banana family discussed in the previous section, we will be rather brief and only highlight the main points. The equal-mass sunrise family has three master integrals. One of these master integrals can be chosen as the tadpole integral $S_{2,2,0,0,0}\left(p^{2}, m^{2} ; 2-2 \epsilon\right)$, which equals one in our normalisation (cf. eq. (2.5)).

Following ref. [22], we choose the remaining two master integrals as ${ }^{1}$

$$
\begin{aligned}
\mathcal{S}_{1}(\epsilon ; t)= & -S_{1,1,1,0,0}\left(p^{2}, m^{2} ; 2-2 \epsilon\right), \\
\mathcal{S}_{2}(\epsilon ; t)= & -\left[\frac{1}{3}\left(t^{2}-6 t+21\right)-12 \epsilon(t-1)\right] S_{1,1,1,0,0}\left(p^{2}, m^{2} ; 2-2 \epsilon\right) \\
& -2(t-1)(t-9) S_{2,1,1,0,0}\left(p^{2}, m^{2} ; 2-2 \epsilon\right),
\end{aligned}
$$

where we encode the kinematics in the dimensionless variable $t=p^{2} / \mathrm{m}^{2}$. Note that the variable $t$ should not be confused with the variable $x$ defined in eq. (2.3) for the banana graph: the two quantities are not trivially related. The precise relation between the quantity $x$ for the banana family and the kinematical variable $t$ defined here will be discussed below.

Just like for the banana family, we will put $m=1$ in the following, as its dependence can be restored later on by simple dimensional analysis. The master integrals in eq. (3.2) satisfy the following system of differential equations [22],

$$
\partial_{t}\left(\begin{array}{l}
\mathcal{S}_{1}(\epsilon ; t) \\
\mathcal{S}_{2}(\epsilon ; t)
\end{array}\right)=(\tilde{B}(t)-2 \epsilon \tilde{D}(t))\left(\begin{array}{l}
\mathcal{S}_{1}(\epsilon ; t) \\
\mathcal{S}_{2}(\epsilon ; t)
\end{array}\right)+\left(\begin{array}{l}
0 \\
1
\end{array}\right)
$$

where $\tilde{B}(t)$ and $\tilde{D}(t)$ are $2 \times 2$ matrices which are independent of $\epsilon$, while the inhomogeneous term comes from the tadpole master integral which decouples from the system of differential equations. For simplicity, in the following we focus on the sunrise family in $d=2$ dimensions. The master integrals $\mathcal{S}_{i}(\epsilon ; t)=\mathcal{S}_{i}(t)+\mathcal{O}(\epsilon)$ are finite in two dimensions, so we can let $\epsilon=0$ in eq. (3.3) and ignore the contribution from $\tilde{D}(t)$. The matrix $\tilde{B}(t)$ is given by ref. [22]. Adapted to our conventions, it reads,

$$
\tilde{B}(t)=\frac{1}{6 t(t-1)(t-9)}\left(\begin{array}{cc}
3\left(3+14 t-t^{2}\right) & -9 \\
(t+3)\left(3+75 t-15 t^{2}+t^{3}\right) & -3\left(3+14 t-t^{2}\right)
\end{array}\right) .
$$

We first have to solve the homogenous equation associated to eq. (3.3), i.e., we need to find a $2 \times 2$ matrix $\mathcal{W}_{S}(t)$ that satisfies $\partial_{t} \mathcal{W}_{S}(t)=\tilde{B}(t) \mathcal{W}_{S}(t)$. The solution to the inhomogeneous equation for $\epsilon=0$ in eq. (3.3) is then obtained by defining the new basis $\left(\mathcal{S}_{1}(t), \mathcal{S}_{2}(t)\right)^{T}=\mathcal{W}_{S}(t)\left(\mathcal{T}_{1}(t), \mathcal{T}_{2}(t)\right)^{T}$ which fulfils the simpler inhomogeneous differential equation,

$$
\partial_{t}\left(\begin{array}{c}
\mathcal{T}_{1}(t) \\
\mathcal{T}_{2}(t)
\end{array}\right)=\mathcal{W}_{S}(t)^{-1}\left(\begin{array}{l}
0 \\
1
\end{array}\right)
$$

\footnotetext{
${ }^{1}$ In ref. [22], the master integrals $\mathcal{S}_{1}$ and $\mathcal{S}_{2}$ are named $g_{6}$ and $g_{7}$ and are defined in eq. (7.7). The kinematical parameter $u$ in [22] equals our parameter $t$, after taking into account that propagators are Euclidean in the definition (5.1) of the integrals in that reference.
} 
The $2 \times 2$ system satisfied by $\mathcal{W}_{S}(t)$ is equivalent to a linear second-order differential equation for the functions in the first row of $\mathcal{W}_{S}(t)[7]$ :

$$
\mathcal{L}_{t}^{(2)}=\partial_{t}^{2}+\left(\frac{1}{t-9}+\frac{1}{t-1}+\frac{1}{t}\right) \partial_{t}+\left(\frac{1}{12(t-9)}+\frac{1}{4(t-1)}-\frac{1}{3 t}\right)
$$

We choose its kernel to be spanned ${ }^{2}$ by the functions $\Psi_{1}$ and $\Psi_{2}$ :

$$
\begin{aligned}
& \Psi_{1}(t)=\frac{4}{\left[(3-\sqrt{t})(1+\sqrt{t})^{3}\right]^{1 / 2}} K\left(\frac{t_{14}(t) t_{23}(t)}{t_{13}(t) t_{24}(t)}\right), \\
& \Psi_{2}(t)=\frac{4 i}{\left[(3-\sqrt{t})(1+\sqrt{t})^{3}\right]^{1 / 2}} K\left(\frac{t_{12}(t) t_{34}(t)}{t_{13}(t) t_{24}(t)}\right),
\end{aligned}
$$

with $t_{i j}(t)=t_{i}(t)-t_{j}(t)$ and

$$
t_{1}(t)=-4, \quad t_{2}(t)=-(1+\sqrt{t})^{2}, \quad t_{3}(t)=-(1-\sqrt{t})^{2}, \quad t_{4}(t)=0 .
$$

The period matrix for the sunrise differential equation is then,

$$
\mathcal{W}_{S}(t)=\left(\begin{array}{cc}
\Psi_{1}(t) & \Psi_{2}(t) \\
\mathcal{D}_{t} \Psi_{1}(t) & \mathcal{D}_{t} \Psi_{2}(t)
\end{array}\right),
$$

with $\mathcal{D}_{t}=\frac{1}{3}\left(3+14 t-t^{2}\right)-\frac{2}{3} t(t-9)(t-1) \partial_{t}$. Note that $\Psi_{1}(t)$ and $\Psi_{2}(t)$ are naturally related to the maximal cut of the integral $S_{1,1,1,0,0}\left(p, m^{2} ; 2\right)$ [7]. It turns out that the second-order differential operator $\mathcal{L}_{t}^{(2)}$ in eq. (3.6) is closely related to the second-order operator $\mathcal{L}_{x}^{(2)}$ for the banana graph provided in eq. (2.19). Indeed, relating the kinematical variables for the sunrise and the banana graph via

$$
x(t)=\frac{-4 t}{(t-1)(t-9)},
$$

one finds

$$
\mathcal{L}_{x}^{(2)}=\tilde{\mathcal{L}}_{t}^{(2)}=\partial_{t}^{2}+\left(\frac{1}{t-9}+\frac{1}{t-1}\right) \partial_{t}+\left(\frac{1}{36(t-9)}-\frac{1}{4(t-1)}+\frac{1}{4 t^{2}}+\frac{2}{9 t}\right) .
$$

This is not quite the same operator as in eq. (3.6). However, one can verify that

$$
\tilde{\mathcal{L}}_{t}^{(2)} \sqrt{t} \Psi_{1}(t)=\tilde{\mathcal{L}}_{t}^{(2)} \sqrt{t} \Psi_{2}(t)=0
$$

that is, the solutions to the two differential operators differ by a square root of $t$.

In section 2.3 we stated that the third-order differential operator $\mathcal{L}_{x}^{(3)}$ in eq. (2.17) is the symmetric square of $\mathcal{L}_{x}^{(2)}$. Correspondingly, the solutions of $\mathcal{L}_{x}^{(3)}$ are sums of products of the functions in eq. (3.7) with an additional factor of $(\sqrt{t})^{2}=t$ which can be precisely traced back to eq. (3.12). In particular, it is straightforward to check that the functions in eq. (2.15) can be cast in the following alternative form which makes manifest the connection

\footnotetext{
${ }^{2}$ In terms of the solutions in ref. [22] one finds that for the region $0<t<1$ we can relate the solutions as $\Psi_{1}(t)=2 I_{1}^{(0,1)}(t)$ and $\Psi_{2}(t)=2 i J_{1}^{(0,1)}(t)$, where the integrals on the right-hand side are defined in eq. (D.11) in ref. [22].
} 
between the fundamental solution matrix for the banana graph, $\mathcal{W}(x)$, and the one for the sunrise, $\mathcal{W}_{S}(t)$, namely

$$
\begin{aligned}
H_{1}(x(t)) & =-\frac{1}{3} t \Psi_{1}(t)^{2} \\
J_{1}(x(t)) & =\frac{i}{3} t \Psi_{1}(t)\left(\Psi_{1}(t)+\Psi_{2}(t)\right), \\
I_{1}(x(t)) & =\frac{1}{3} t\left(\Psi_{1}(t)+\Psi_{2}(t)\right)\left(\Psi_{1}(t)+3 \Psi_{2}(t)\right) .
\end{aligned}
$$

We see that, as expected, the solutions of $\mathcal{L}_{x}^{(3)}$ are sums of products of the solutions of $\mathcal{L}_{x}^{(2)}$ with an additional prefactor of $t$.

Equation (3.13) is our first hint that the function spaces of the sunrise and banana families in $d=2$ dimensions are closely related. Since the two-loop sunrise graph can be expressed in terms of elliptic polylogarithms [17-21, 33, 76] and iterated integrals of modular forms [35, 46], it is tantalising to investigate whether the same class of functions describes the banana family in $d=2$ dimensions as well. This was already hinted at in ref. [51], where it was argued that the three-loop equal-mass banana graph is an elliptic trilogarithm and closely related to the same congruence subgroup relevant to the two-loop equal-mass sunrise graph. In the remainder of this paper we make this connection concrete, and we present analytic results for the equal-mass banana graph in $d=2$ dimensions in terms of the same class of functions as for the two-loop equal-mass sunrise graph.

\subsection{The elliptic curve associated to the sunrise graph}

Since the goal of this paper is to show that the equal-mass sunrise and banana graphs can be expressed in terms of the same class of functions, let us review in the remainder of this section the geometric objects and functions that appear in the computation of the two-loop equal-mass sunrise graph.

In the previous section we have seen that the homogeneous solutions of the second order differential equation satisfied by the two-loop equal-mass sunrise graph can be expressed in terms complete elliptic integrals of the first kind, cf. eq. (3.7). The appearance of complete elliptic integrals is closely related to the presence of an elliptic curve in the geometry associated to the problem. Loosely speaking, an elliptic curve can be defined as the set of points $(x, y)$ that solve the polynomial equation $y^{2}=\left(x-a_{1}\right) \cdots\left(x-a_{4}\right)$, where the $a_{i}$ are complex numbers that are constants with respect to $(x, y)$. Instead of characterising an elliptic curve by the roots $a_{i}$ of the polynomial equation, we can also characterise it by its two periods, defined by

$$
\omega_{1}=2 \mathrm{~K}(\lambda) \text { and } \omega_{2}=2 i \mathrm{~K}(1-\lambda) \text {, with } \lambda=\frac{\left(a_{1}-a_{4}\right)\left(a_{2}-a_{3}\right)}{\left(a_{1}-a_{3}\right)\left(a_{2}-a_{4}\right)} .
$$

The periods are not uniquely defined, but we could replace them by any integer linear combination of the $\omega_{1}$ and $\omega_{2}$ chosen above. More precisely, the periods are only defined modulo $\operatorname{SL}(2, \mathbb{Z})$ transformations, which act on the two periods as follows

$$
\left(\begin{array}{l}
\omega_{2} \\
\omega_{1}
\end{array}\right) \rightarrow\left(\begin{array}{ll}
a & b \\
c & d
\end{array}\right)\left(\begin{array}{l}
\omega_{2} \\
\omega_{1}
\end{array}\right), \quad\left(\begin{array}{ll}
a & b \\
c & d
\end{array}\right) \in \mathrm{SL}(2, \mathbb{Z})
$$


Such transformations are called modular transformations. The geometry is also left unchanged by a rescaling, and so only the ratio of the two periods carries relevant information

$$
\tau=\frac{\omega_{2}}{\omega_{1}}=i \frac{\mathrm{K}(1-\lambda)}{\mathrm{K}(\lambda)},
$$

where it is customary to refer to $\tau$ as the modular parameter of the elliptic curve. Modular transformations act on $\tau$ via Möbius transformations,

$$
\tau \rightarrow \frac{a \tau+b}{c \tau+d}, \quad\left(\begin{array}{ll}
a & b \\
c & d
\end{array}\right) \in \operatorname{SL}(2, \mathbb{Z}) .
$$

Note that it is always possible to choose $\tau$ to lie in the complex upper half-plane $\mathbb{H}=\{\tau \in$ $\mathbb{C} \mid \operatorname{Im} \tau>0\}$.

In many situations one is not interested in modular transformations associated with the full group $\mathrm{SL}(2, \mathbb{Z})$, but only a subgroup $\Gamma \subset \mathrm{SL}(2, \mathbb{Z})$ is relevant. In particular, in many applications in mathematics and physics the various congruence subgroups of level $N$ play a prominent role,

$$
\begin{aligned}
\Gamma_{0}(N) & =\left\{\left(\begin{array}{ll}
a & b \\
c & d
\end{array}\right) \in \mathrm{SL}(2, \mathbb{Z}) \mid c \equiv 0 \bmod N\right\}, \\
\Gamma_{1}(N) & =\left\{\left(\begin{array}{ll}
a & b \\
c & d
\end{array}\right) \in \mathrm{SL}(2, \mathbb{Z}) \mid a, d \equiv 1 \bmod N \text { and } c \equiv 0 \bmod N\right\}, \\
\Gamma(N) & =\left\{\left(\begin{array}{ll}
a & b \\
c & d
\end{array}\right) \in \mathrm{SL}(2, \mathbb{Z}) \mid a, d \equiv 1 \bmod N \text { and } b, c \equiv 0 \bmod N\right\} .
\end{aligned}
$$

Let us now discuss how a family of elliptic curves arises from the sunrise graph. We see from eq. (3.14) that the periods of an elliptic curve can be expressed in terms of complete elliptic integrals of the first kind. The same is true for the functions $\Psi_{1}(t)$ and $\Psi_{2}(t)$, which define two independent periods of a family of elliptic curves parametrised by the parameter $t$. The polynomial equation describing a member of this family is $y^{2}=$ $\left(x-t_{1}(t)\right) \ldots\left(x-t_{4}(t)\right)$, where the $t_{i}(t)$ were defined in eq. (3.8). A member of this family can be defined equivalently by specifying the value of $t$ or of the modular parameter $\tau$,

$$
\tau=\frac{\Psi_{2}(t)}{\Psi_{1}(t)} .
$$

It is possible to invert eq. (3.19) and express $t$ as a function of $\tau$ [77],

$$
t(\tau)=9 \frac{\eta(\tau)^{4} \eta(6 \tau)^{8}}{\eta(2 \tau)^{8} \eta(3 \tau)^{4}}
$$

where $\eta(\tau)$ denotes the Dedekind $\eta$-function,

$$
\eta(\tau)=q^{1 / 24} \prod_{n=1}^{\infty}\left(1-q^{n}\right), \quad q=e^{2 \pi i \tau} .
$$

The function $t(\tau)$ is invariant under modular transformations for $\Gamma_{1}(6)$,

$$
t\left(\frac{a \tau+b}{c \tau+d}\right)=t(\tau), \quad\left(\begin{array}{ll}
a & b \\
c & d
\end{array}\right) \in \Gamma_{1}(6) .
$$


Therefore, the family of elliptic curves associated to the sunrise graph is tightly related to the congruence subgroup $\Gamma_{1}(6)[17,46] .^{3}$

In general, we need to consider not only functions that are invariant under $\Gamma \subset \operatorname{SL}(2, \mathbb{Z})$, but also functions with non-trivial transformation behaviour. A modular form of weight $n$ for $\Gamma$ is a holomorphic function $f$ which transforms covariantly under modular transformations for the group $\Gamma$,

$$
f\left(\frac{a \tau+b}{c \tau+d}\right)=(c \tau+d)^{n} f(\tau), \quad\left(\begin{array}{ll}
a & b \\
c & d
\end{array}\right) \in \Gamma,
$$

subject to some regularity conditions which we can ignore at this point. It is easy to see that modular forms define an algebra: the product of two modular forms of weights $n_{1}$ and $n_{2}$ is a modular form of weight $n_{1}+n_{2}$. If we denote by $\mathcal{M}_{n}(\Gamma)$ the vector space of modular forms of weight $n$ for $\Gamma$, then $\mathcal{M}_{n}(\Gamma)$ is always finite-dimensional. It is possible to construct bases for $\mathcal{M}_{n}(\Gamma)$ in a completely algorithmic way. Here we only discuss the case $\Gamma=\Gamma_{1}(6)$, which is relevant to the computation of the equal-mass sunrise and banana graphs. The basis described below was introduced in ref. [78].

We start by noting that the function

$$
f_{1,0}(\tau)=\Psi_{1}(t(\tau))
$$

is a modular form of weight one for $\Gamma_{1}(6)[46,78]$. Since modular forms form an algebra, it is clear that $\left(f_{1,0}(\tau)\right)^{n}$ will define a modular form of weight $n$. Moreover, since $t(\tau)$ in eq. (3.20) is invariant under $\Gamma_{1}(6)$, multiplying powers of $f_{1,0}(\tau)$ by any (rational) function of $t(\tau)$ will not change the behaviour under modular transformations for $\Gamma_{1}(6)$. The requirement that modular forms be holomorphic everywhere restricts these rational functions to be polynomials. The maximal power of this polynomial can be constrained by analysing the behaviour of $\Psi_{1}(t)$ for large values of $t$ (for details see ref. [78]). With these considerations, one finds that a basis of $\mathcal{M}_{n}\left(\Gamma_{1}(6)\right)$ is given by the functions [78]

$$
f_{n, p}(\tau)=\Psi_{1}(t(\tau))^{n} t(\tau)^{p}, \quad 0 \leq p \leq n .
$$

Note that this definition extends to modular forms of weight zero, $f_{0,0}(\tau)=1$. The advantage of this basis in the context of the sunrise and banana graphs will be discussed in the remainder of this section.

\subsection{A class of iterated integrals of modular forms for $\Gamma_{1}(6)$}

After this excursion into the geometry associated to the sunrise graph, let us now review what it can teach us about the functions the sunrise graph evaluates to. It is known that the two-loop equal-mass sunrise integral can be expressed in terms of iterated integrals of modular forms for $\Gamma_{1}(6)$ [46]. In this section we give a short review of these functions with a special focus on the case of $\Gamma_{1}(6)$.

\footnotetext{
${ }^{3}$ Depending on whether one starts from the elliptic curve obtained from the Feynman parameter integral or the maximal cut, one may instead find $\Gamma_{1}(12)$, see ref. [46] for a detailed discussion.
} 
If $f_{i_{a}}(\tau)$ are modular forms of weight $n_{i_{a}}$ for a congruence subgroup $\Gamma$, we define the iterated integrals $[44,45]$

$$
\mathcal{I}\left(f_{i_{1}}, \ldots, f_{i_{k}} ; \tau\right)=\int_{i \infty}^{\tau} d \tau^{\prime} f_{i_{1}}\left(\tau^{\prime}\right) \mathcal{I}\left(f_{i_{2}}, \ldots, f_{i_{k}} ; \tau^{\prime}\right)
$$

In general these integrals may diverge, but the divergences can be regulated in a standard way [45] (see also ref. [46] for a pedagogical introduction). Moreover, these integrals satisfy all the properties of iterated integrals. In particular they form a shuffle algebra. We define the length of $\mathcal{I}\left(f_{i_{1}}, \ldots, f_{i_{k}} ; \tau\right)$ as $k$.

Let us now discuss how we can associate a concept of transcendental weight to the functions $\mathcal{I}\left(f_{i_{1}}, \ldots, f_{i_{k}} ; \tau\right)$. If $\Gamma$ is a congruence subgroup of level $N$, then modular forms for $\Gamma$ are invariant under translations by $N, f_{i_{a}}(\tau+N)=f_{i_{a}}(\tau)$. Hence, $f_{i_{a}}(\tau)$ admits a Fourier series of the form

$$
f_{i_{a}}(\tau)=\sum_{n=0}^{\infty} a_{n} q_{N}^{n}, \quad q_{N}=e^{2 \pi i \tau / N} .
$$

It is always possible to choose a basis such that the Fourier coefficients are rational multiples of $\pi^{n_{i_{a}}}$. With this normalisation, we define the transcendental weight ${ }^{4}$ of $\mathcal{I}\left(f_{i_{1}}, \ldots, f_{i_{k}} ; \tau\right)$ to be $\sum_{a=1}^{k} n_{i_{a}}$. The rationale behind this definition will become clear in the next section.

In the case $\Gamma=\Gamma_{1}(6)$, we can work with the explicit basis of modular forms in eq. (3.25) and we define

$$
\mathcal{I}\left(\begin{array}{ccc}
n_{1} & \ldots & n_{k} \\
p_{1} & \ldots & p_{k}
\end{array} ; \tau\right)=\mathcal{I}\left(f_{n_{1}, p_{1}}, \ldots, f_{n_{k}, p_{k}} ; \tau\right) \text {. }
$$

It is easy to check that the modular forms $f_{n, p}(\tau)$ are normalised such that their Fourier coefficients are proportional to $\pi^{n}$. Hence, $\mathcal{I}\left(\begin{array}{lll}n_{1} & \ldots & n_{k} \\ p_{1} & \ldots & p_{k}\end{array} \tau\right)$ has length $k$ and weight $\sum_{a=1}^{k} n_{a}$.

The iterated integrals $\mathcal{I}\left(\begin{array}{ccc}n_{1} & \ldots & n_{k} \\ p_{1} & \ldots & p_{k}\end{array} ;\right)$ have an important property: they allow for an alternative description in terms of iterated integrals over products of complete elliptic integrals, similar to those that have appeared in refs. $[22,57]$ in the context of the sunrise and banana graphs. The basic idea is the following: we see from eq. (3.25) that if we change variables from $\tau$ to $t$ using eq. (3.19), then $f_{n, p}(\tau)$ is proportional to $\Psi_{1}(t)^{n}$. The Jacobian of the change of variables is given by

$$
d \tau=-\frac{6 \pi i d t}{t(t-1)(t-9) \Psi_{1}(t)^{2}},
$$

where we used the fact that

$$
\operatorname{det} \mathcal{W}_{S}(t)=-\frac{2}{3} t(t-9)(t-1)\left[\Psi_{1}(t) \partial_{t} \Psi_{2}(t)-\Psi_{2}(t) \partial_{t} \Psi_{1}(t)\right]=4 \pi i
$$

Hence, the integration kernels that define the iterated integrals can be cast in the form

$$
d \tau f_{n, p}(\tau)=-\frac{6 \pi i d t t^{p-1}}{(t-1)(t-9)} \Psi_{1}(t)^{n-2},
$$

\footnotetext{
${ }^{4}$ Note that the transcendental weight of the iterated integrals is distinct from the weight of a modular form under modular transformations. In particular, the iterated integrals will in general not be modular forms.
} 
and in this way we obtain an alternative description of the iterated integrals for $\Gamma_{1}(6)$ as iterated integrals over products of complete elliptic integrals,

$$
\mathcal{I}\left(\begin{array}{cccc}
n_{1} & \ldots & n_{k} \\
p_{1} & \ldots & p_{k}
\end{array} ; \tau\right)=-6 \pi i \int_{0}^{t(\tau)} \frac{d t^{\prime} t^{\prime p_{1}-1}}{\left(t^{\prime}-1\right)\left(t^{\prime}-9\right)} \Psi_{1}\left(t^{\prime}\right)^{n_{1}-2} \mathcal{I}\left(\begin{array}{cccc}
n_{2} & \ldots & n_{k} \\
p_{2} & \ldots & p_{k}
\end{array} \tau^{\prime}\left(t^{\prime}\right)\right) .
$$

We see that the basis of modular forms in eq. (3.25) and the iterated integrals in eq. (3.28) allow us to easily switch between the two representations in terms of modular forms or products of complete elliptic integrals. This observation will be the key to expressing the master integrals for the banana family as iterated integrals of modular forms for $\Gamma_{1}(6)$. Before we do this, we find it instructive to review the same procedure in the context of the master integrals for the sunrise family.

\subsection{The sunrise integral and modular forms for $\Gamma_{1}(6)$}

To see how the two-loop sunrise integral can be expressed in terms of iterated integrals of modular forms, we start from the differential equation in eq. (3.5), which we rewrite as

$$
\partial_{t}\left(\begin{array}{l}
\mathcal{T}_{1}(t) \\
\mathcal{T}_{2}(t)
\end{array}\right)=\frac{1}{4 \pi i}\left(\begin{array}{r}
-\Psi_{2}(t) \\
\Psi_{1}(t)
\end{array}\right) .
$$

We change variables from $t$ to the modular parameter $\tau$ using eq. (3.20). The Jacobian of the change of variables can easily be read of from eq. (3.29). We find

$$
\partial_{\tau}=-\frac{1}{6 \pi i} t(\tau)(t(\tau)-1)(t(\tau)-9) \Psi_{1}(t(\tau))^{2} \partial_{t},
$$

and so eq. (3.33) becomes

$$
\begin{aligned}
\partial_{\tau}\left(\begin{array}{c}
\mathcal{T}_{1}(t(\tau)) \\
\mathcal{T}_{2}(t(\tau))
\end{array}\right) & =\frac{1}{24 \pi^{2}} t(\tau)(t(\tau)-1)(t(\tau)-9) \Psi_{1}(t(\tau))^{2}\left(\begin{array}{r}
-\Psi_{2}(t) \\
\Psi_{1}(t)
\end{array}\right) \\
& =\frac{1}{24 \pi^{2}}\left(f_{3,3}(\tau)-10 f_{3,2}(\tau)+9 f_{3,1}(\tau)\right)\left(\begin{array}{c}
-\tau \\
1
\end{array}\right) \\
& =\frac{1}{24 \pi^{2}}\left(f_{3,3}(\tau)-10 f_{3,2}(\tau)+9 f_{3,1}(\tau)\right)\left(\begin{array}{c}
-\mathcal{I}\left(\begin{array}{c}
0 \\
0
\end{array} ;\right) \\
1
\end{array}\right),
\end{aligned}
$$

where we used the fact that

$$
\tau=\int_{i \infty}^{\tau} d \tau^{\prime} f_{0,0}\left(\tau^{\prime}\right)=\mathcal{I}\left({ }_{0}^{0} ; \tau\right) .
$$

We can choose as initial condition the point $t=0$, which corresponds to $\tau \rightarrow i \infty$. Translating the results of ref. [22] to our conventions we find

$$
\begin{aligned}
& \mathcal{T}_{1}(t)=\mathrm{Cl}_{2}(\pi / 3)+\mathcal{O}(t), \\
& \mathcal{T}_{2}(t)=0+\mathcal{O}(t) .
\end{aligned}
$$

Here $\mathrm{Cl}_{2}(x)$ denotes the Clausen function,

$$
\mathrm{Cl}_{2}(x)=\frac{i}{2}\left(\operatorname{Li}_{2}\left(e^{-i x}\right)-\mathrm{Li}_{2}\left(e^{i x}\right)\right) .
$$


We then find the following result for $\mathcal{T}_{i}$,

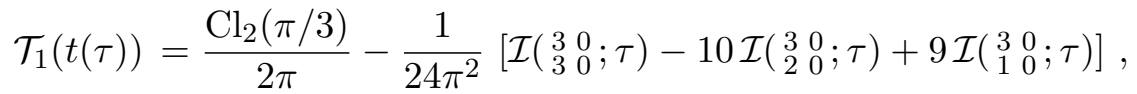

$$
\begin{aligned}
& \mathcal{T}_{2}(t(\tau))=\frac{1}{24 \pi^{2}}\left[\mathcal{I}\left({ }_{3}^{3} ; \tau\right)-10 \mathcal{I}\left({ }_{2}^{3} ; \tau\right)+9 \mathcal{I}\left({ }_{1}^{3} ; \tau\right)\right] .
\end{aligned}
$$

Let us make a comment about the form of the result for the sunrise graph in eq. (3.39). It is easy to see that the result in eq. (3.39) is a linear combination of functions of uniform weight one, where the weight of the iterated integrals of modular forms was defined earlier, and the weight of Clausen function and $\pi$ is defined in the usual way. This fact was first observed in ref. [36].

\section{Analytic results for the equal-mass banana graph}

After a brief detour through the sunrise integral family, in this section we return to the banana family and present the main results of this paper. We derive fully analytic results for all master integrals for the equal-mass banana graphs in $d=2$ dimensions. In order to achieve this, we proceed in exactly the same way as for the sunrise graph in the previous section: we start by showing how we can relate the fundamental solution matrix of the system of differential equations satisfied by the master integrals for the banana family, eq. (2.11), to modular forms for $\Gamma_{1}(6)$. In particular, we express the results for all master integrals in terms of the iterated integrals of modular forms for $\Gamma_{1}(6)$ defined earlier in eq. (3.28).

After representing the equal-mass banana integral in terms of modular forms for $\Gamma_{1}(6)$, we carry on with the main theme of this paper and ask whether the banana integral can also be recast in terms of other functions used in the past to represent the sunrise integral [46]. We then show the result for the banana integral in terms of iterated integrals of Eisenstein series of level six and elliptic multiple polylogarithms (eMPLs).

\subsection{The equal-mass banana graph and modular forms for $\Gamma_{1}(6)$}

We start from eq. (3.13), which relates the entry $H_{1}(x)$ in the fundamental solution matrix $\mathcal{W}(x)$ to the maximal cut of the equal-mass sunrise graph in $d=2$ dimensions. Comparing eqs. (3.13) and (3.25), we immediately see that

$$
\begin{aligned}
H_{1}(x(\tau)) & =-\frac{1}{3} f_{2,1}(\tau), \\
J_{1}(x(\tau)) & =\frac{i}{3} f_{2,1}(\tau)(1+\tau), \\
I_{1}(x(\tau)) & =\frac{1}{3} f_{2,1}(\tau)(1+\tau)(1+3 \tau) .
\end{aligned}
$$

where $x(\tau)$ is obtained by composing eq. (3.10) with eq. (3.20), and can be written as $[51,56]$

$$
x(\tau)=-4\left(\frac{\eta(2 \tau) \eta(6 \tau)}{\eta(\tau) \eta(3 \tau)}\right)^{6} .
$$

We see that after changing variables from $x$ to $\tau, H_{1}(x(\tau))$ is a modular form of weight two for $\Gamma_{1}(6)$, while $J_{1}(x(\tau))$ and $I_{1}(x(\tau))$ are modular forms multiplied by a polynomial 
in $\tau$. The other entries in $\mathcal{W}(x)$ also involve derivatives of $\Psi_{1}(t)$ and $\Psi_{2}(t)$, and so they cannot be expressed in terms of $f_{2,1}(\tau)$ and $\tau$ alone.

In a next step, let us rewrite the differential equations for the three master integrals of the banana graph, eq. (2.14), in terms of the modular parameter $\tau$ and express them in the language of modular forms for $\Gamma_{1}(6)$. In order to do so, we need to include the Jacobian from the change of variables from $x$ to $\tau$, whose value is easily obtained by combining eq. (3.10) with eq. (3.29). We find,

$$
\partial_{\tau}=-\frac{2 t(\tau)\left(t(\tau)^{2}-9\right)}{3 \pi i(t(\tau)-1)(t(\tau)-9)} \Psi_{1}(t(\tau))^{2} \partial_{x}
$$

The desired differential equation immediately follows upon expressing the elements of the fundamental solution matrix $\mathcal{W}(x)$ in terms of the basis of modular forms for $\Gamma_{1}(6)$ in eq. (3.25) and inverting it. Note that the expression for $\mathcal{W}(x)^{-1}$ involves derivatives of $\Psi_{1}(t)$ and $\Psi_{2}(t)$, but we find that the dependence on the derivatives drops out once eq. (3.30) is imposed. Combining everything, we find

$$
\begin{aligned}
\partial_{\tau}\left(\begin{array}{l}
M_{1}(x(\tau)) \\
M_{2}(x(\tau)) \\
M_{3}(x(\tau))
\end{array}\right) & =\frac{f_{4,4}(\tau)-10 f_{4,3}(\tau)+90 f_{4,1}(\tau)-81 f_{4,0}(\tau)}{18 i \pi^{3}}\left(\begin{array}{c}
3(1+\tau)^{2} \\
-2 i(2+3 \tau) \\
-1
\end{array}\right) \\
& =\frac{f_{4,4}(\tau)-10 f_{4,3}(\tau)+90 f_{4,1}(\tau)-81 f_{4,0}(\tau)}{18 i \pi^{3}}\left(\begin{array}{c}
3\left(1+\mathcal{I}\left(\begin{array}{l}
0 \\
0
\end{array} ; \tau\right)\right)^{2} \\
-2 i\left(2+3 \mathcal{I}\left({ }_{0}^{0} ; \tau\right)\right) \\
-1
\end{array}\right) .
\end{aligned}
$$

The above differential equation can be solved by quadrature using the iterated integrals defined in eq. (3.28). The initial condition of the differential equation can be obtained by analysing the behaviour of the master integrals $\mathcal{I}_{i}(x)$ in eq. (2.4) as $x \rightarrow 0$, which corresponds to $\tau \rightarrow i \infty$, the lower integration limit of the iterated integrals in eq. (3.26). We find,

$$
\begin{aligned}
& \mathcal{I}_{1}(x)=x \log ^{3}(-x / 4)-4 x \zeta_{3}+\mathcal{O}\left(x^{2}\right), \\
& \mathcal{I}_{2}(x)=\frac{3}{4} x \log ^{2}(-x / 4)+\mathcal{O}\left(x^{2}\right), \\
& \mathcal{I}_{3}(x)=\frac{1}{2} x \log (-x / 4)+\mathcal{O}\left(x^{2}\right) .
\end{aligned}
$$

The derivation of eq. (4.5) is straightforward but technical. We refer to appendix A for the details. Putting everything together, we find the following result for the master integrals of the equal-mass banana family in $d=2$ dimensions,

$$
\begin{aligned}
& M_{1}(x(\tau))=-\frac{4 \zeta_{3}}{\pi^{2}}-\frac{i}{6 \pi^{3}}\left[81 \mathcal{I}\left({ }_{0}^{4} ; \tau\right)-90 \mathcal{I}\left({ }_{1}^{4} ; \tau\right)+10 \mathcal{I}\left({ }_{3}^{4} ; \tau\right)-\mathcal{I}\left({ }_{4}^{4} ; \tau\right)\right.
\end{aligned}
$$

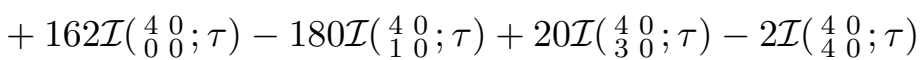

$$
\begin{aligned}
& \left.+162 \mathcal{I}\left(\begin{array}{ccc}
4 & 0 & 0 \\
0 & 0 & 0
\end{array} ;\right)-180 \mathcal{I}\left(\begin{array}{ccc}
4 & 0 & 0 \\
1 & 0 & 0
\end{array} ;\right)+20 \mathcal{I}\left(\begin{array}{ccc}
4 & 0 & 0 \\
3 & 0 & 0
\end{array} ;\right)-2 \mathcal{I}\left(\begin{array}{ccc}
4 & 0 & 0 \\
4 & 0 & 0
\end{array} ;\right)\right] \text {, } \\
& M_{2}(x(\tau))=-\frac{1}{9 \pi^{3}}\left[162 \mathcal{I}\left({ }_{0}^{4} ; \tau\right)-180 \mathcal{I}\left({ }_{1}^{4} ; \tau\right)+20 \mathcal{I}\left({ }_{3}^{4} ; \tau\right)-2 \mathcal{I}\left({ }_{4}^{4} ; \tau\right)\right.
\end{aligned}
$$

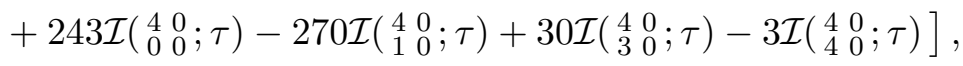

$$
\begin{aligned}
& M_{3}(x(\tau))=\frac{i}{18 \pi^{3}}\left[81 \mathcal{I}\left({ }_{0}^{4} ; \tau\right)-90 \mathcal{I}\left({ }_{1}^{4} ; \tau\right)+10 \mathcal{I}\left({ }_{3}^{4} ; \tau\right)-\mathcal{I}\left({ }_{4}^{4} ; \tau\right)\right] \text {. }
\end{aligned}
$$


We note again that the integrals $\mathcal{I}\left(\begin{array}{l}n \\ 0\end{array} ;\right)$ are formally logarithmically divergent for $\tau \rightarrow i \infty$. However, all divergences can be subtracted and shuffled out in the standard way such that they are captured solely in terms of powers of $\mathcal{I}\left({ }_{0}^{0} ; \tau\right)=\tau$. After regularisation, all iterated integrals of modular forms can be evaluated numerically with high precision, and we have checked that eq. (4.6) numerically agrees with a direct numerical evaluation of the corresponding Feynman parameter representation.

We can see that, just like the result for the sunrise integral in eq. (3.39), our results in eq. (4.6) have uniform weight one. Unlike the sunrise result, however, the expressions for $M_{i}$ in eq. (4.6) do not have uniform length, i.e. they are composed of iterated integrals with numbers of integrations ranging from one to three. It is possible to perform a change of basis which casts the result as integrals which have both uniform length and weight. In order to achieve this, we decompose the fundamental solution $\mathcal{W}$ into a semi-simple times a unipotent matrix,

$$
\mathcal{W}=S U \text {. }
$$

An additional motivation to split the homogeneous solution into a semi-simple and a unipotent part comes from ref. [36], where it was argued that this splitting naturally leads to Feynman integrals of uniform weight. An algorithmic way to construct this splitting in the present is described in appendix B. Given the solution matrix $\mathcal{W}$ and using eq. (3.19), we can find the unipotent matrix

$$
\begin{aligned}
U & =\left(\begin{array}{ccc}
1 & -\frac{\left.i \Psi_{1}(t)+\Psi_{2}(t)\right)}{\Psi_{1}(t)} & -\frac{\left(\Psi_{1}(t)+\Psi_{2}(t)\right)\left(\Psi_{1}(t)+3 \Psi_{2}(t)\right)}{\Psi_{1}(t)^{2}} \\
0 & 1 & -\frac{2 i\left(2 \Psi_{1}(t)+3 \Psi_{2}(t)\right)}{\Psi_{1}(t)} \\
0 & 0 & 1
\end{array}\right) \\
& =\left(\begin{array}{ccc}
1 & -i(\tau+1) & -(\tau+1)(3 \tau+1) \\
0 & 1 & -2 i(3 \tau+2) \\
0 & 0 & 1
\end{array}\right) .
\end{aligned}
$$

Using this decomposition, we find that

$$
\mathcal{W}(x(\tau))\left(\begin{array}{l}
M_{1}(x(\tau)) \\
M_{2}(x(\tau)) \\
M_{3}(x(\tau))
\end{array}\right)=S(\tau)\left(\begin{array}{l}
\tilde{M}_{1}(x(\tau)) \\
\tilde{M}_{2}(x(\tau)) \\
\tilde{M}_{3}(x(\tau))
\end{array}\right),
$$

with

$$
\left(\begin{array}{l}
\tilde{M}_{1}(x(\tau)) \\
\tilde{M}_{2}(x(\tau)) \\
\tilde{M}_{3}(x(\tau))
\end{array}\right)=U(\tau)\left(\begin{array}{l}
M_{1}(x(\tau)) \\
M_{2}(x(\tau)) \\
M_{3}(x(\tau))
\end{array}\right) .
$$

The functions $\tilde{M}_{i}$ are of uniform weight two and of uniform length, given by

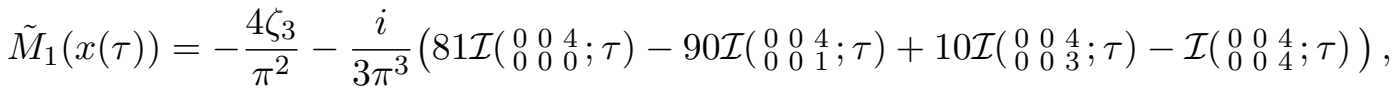

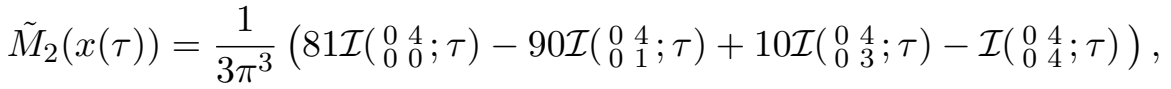

$$
\begin{aligned}
& \tilde{M}_{3}(x(\tau))=\frac{i}{18 \pi^{3}}\left(81 \mathcal{I}\left({ }_{0}^{4} ; \tau\right)-90 \mathcal{I}\left({ }_{1}^{4} ; \tau\right)+10 \mathcal{I}\left({ }_{3}^{4} ; \tau\right)-\mathcal{I}\left({ }_{4}^{4} ; \tau\right)\right) \text {. }
\end{aligned}
$$


We can further see that the three different solutions $\tilde{M}_{i}$ are related to each other by taking $\tau$-derivatives:

$$
\begin{aligned}
& \tilde{M}_{2}(x(\tau))=i \partial_{\tau} \tilde{M}_{1}(x(\tau)) \\
& \tilde{M}_{3}(x(\tau))=\frac{i}{6} \partial_{\tau} \tilde{M}_{2}(x(\tau))=-\frac{1}{6} \partial_{\tau}^{2} \tilde{M}_{1}(x(\tau)) .
\end{aligned}
$$

In eq. (4.11) we have expressed the banana integral family in terms of iterated integrals of the same modular forms already encountered in the sunrise case. It is worth exploring whether we can also represent the solution in terms of another class of functions which play a prominent role in the analytic calculation of the two-loop sunrise graph: elliptic multiple polylogarithms (eMPLs). In the following we show how we can recast eq. (4.11) in terms of these functions.

\subsection{Solution in terms of elliptic polylogarithms}

In the previous section, we saw how the banana integral can be expressed in terms of iterated integrals over the homogeneous solution of the sunrise integral. The sunrise integral itself has been computed in many different forms before. Here, we are particularly interested in the fact that the sunrise integral can also be represented in terms of elliptic polylogarithms [33]. It is therefore natural to ask the question whether it is possible to express the banana integral in terms of elliptic polylogarithms as well. To answer this question, let us recall the definition of the eMPLs as used in ref. [33] (see also ref. [41]),

$$
\tilde{\Gamma}\left(\begin{array}{cccc}
n_{1} & \ldots & n_{k} \\
z_{1} & \ldots & z_{k}
\end{array} ; z_{k+1}, \tau\right)=\int_{0}^{z_{k+1}} d w g^{\left(n_{1}\right)}\left(w-z_{1} ; \tau\right) \tilde{\Gamma}\left(\begin{array}{ccccc}
n_{2} & \ldots & n_{k} \\
z_{2} & \ldots & z_{k}
\end{array} ; w, \tau\right) .
$$

Here the integration kernels $g^{(n)}(z ; \tau)$ are related to expansion coefficients of the EisensteinKronecker series as defined in ref. [33]. The exact form of these kernels is immaterial for the following arguments, though it is important to note that for $z=\frac{r}{N}+\frac{s}{N} \tau$, with $r, s \in \mathbb{Z}$ and $N \in \mathbb{N}$ the integration kernels $g$ can be expressed as

$$
g^{(n)}\left(\frac{r}{N}+\frac{s}{N} \tau, \tau\right)=\sum_{k=0}^{n} \frac{\left(2 \pi i \frac{s}{N}\right)^{k}}{k !} h_{N, r, s}^{(n-k)}(\tau),
$$

where the functions $h_{N, r, s}^{(n-k)}, 0 \leq r, s<N$, denote modular forms of weight $k>1$ for $\Gamma(N)$ (cf. eq. (3.18)) defined as [35]

$$
h_{N, r, s}^{(k)}(\tau)=-\sum_{\substack{(\alpha, \beta) \in \mathbb{Z}^{2} \\(\alpha, \beta) \neq(0,0)}} \frac{e^{2 \pi i(s \alpha-r \beta) / N}}{(\alpha+\beta \tau)^{2 n}} .
$$

Not all these Eisenstein series are linearly independent. In ref. [35] it was shown that the Eisenstein series of weight $k \geq 2$ for $\Gamma(N)$ are spanned by the set $\left\{h_{N, r, s}^{(k)}\right\}_{0 \leq r, s<N}$.

From this observation it follows that whenever all the arguments of an eMPL are rational points, $z_{i}=\frac{r_{i}}{N}+\tau \frac{s_{i}}{N}$, then this function can be written as a linear combination of Eisenstein series for $\Gamma(N)$, defined as

$$
\begin{aligned}
I\left(\begin{array}{cc}
n_{1} & N_{1} \\
r_{1} & s_{1}
\end{array}|\ldots| \begin{array}{ll}
n_{k} & N_{k} \\
r_{k} & s_{k}
\end{array} ; \tau\right) & \equiv I\left(h_{N_{1}, r_{1}, s_{1}}^{\left(n_{1}\right)}, \ldots, h_{N_{k}, r_{k}, s_{k}}^{\left(n_{k}\right)} ; \tau\right) \\
& =\int_{i \infty}^{\tau} d \tau^{\prime} h_{N_{1}, r_{1}, s_{1}}^{\left(n_{1}\right)}\left(\tau^{\prime}\right) I\left(\begin{array}{cc}
n_{2} & N_{2} \\
r_{2} & s_{2}
\end{array}|\ldots| \begin{array}{cc}
n_{k} & N_{k} \\
r_{k} & s_{k}
\end{array} \tau^{\prime}\right)
\end{aligned}
$$


with $h_{0,0,0}^{(0)}(\tau) \equiv 1$. The converse statement, however, is not always true: not every iterated integral of Eisenstein series for $\Gamma(N)$ can be written in terms of eMPLs evaluated at rational points, but this is only possible for specific combinations of iterated integrals of Eisenstein series (cf., e.g., refs. [45, 48, 79]). The combination of iterated integrals of Eisenstein series that describes the sunrise integrals satisfies this criterion. It is therefore natural to ask if the same holds true for the banana graph. In ref. [51] it was argued that the banana integral with unit powers of the propagators corresponds to an elliptic trilogarithm. In the remainder of this section we make this statement explicit and extend it to the other two master integrals for the banana graph, and we show how the representation in terms of eMPLs can be obtained.

In broad strokes, the strategy we follow is very simple: we write down a very general ansatz of eMPLs of length three with rational arguments with $N=6$. We can express each of these in terms of iterated integrals of Eisenstein series for $\Gamma(6)$ using the techniques described in ref. [35], and we match this expression to our results for the banana integrals from the previous section. At this point, however, we need to make a technical comment: while eMPLs naturally give rise to iterated integrals of Eisenstein series for $\Gamma(6)$, the banana integrals in eq. (4.11) involve Eisenstein series for $\Gamma_{1}(6)$. Matching our ansatz of eMPLs to eq. (4.11) is therefore not completely straightforward. However, since $\Gamma_{1}(6)$ is a subgroup of $\Gamma(6)$, we can express all Eisenstein series for $\Gamma_{1}(6)$ in terms of those for $\Gamma(6)$. In particular, at weight four (which is of relevance here, cf. eq. (4.11)), there are four Eisenstein series for $\Gamma_{1}(6)$, which can be written as linear combinations of the basis of Eisenstein series for $\Gamma(6)$ as follows,

$$
\begin{aligned}
& b_{1}(\tau)=h_{6,0,1}^{(4)}(\tau)+h_{6,1,1}^{(4)}(\tau)+h_{6,2,1}^{(4)}(\tau)+h_{6,3,1}^{(4)}(\tau)+h_{6,4,1}^{(4)}(\tau)+h_{6,5,1}^{(4)}(\tau), \\
& b_{2}(\tau)=h_{6,1,2}^{(4)}(\tau)+h_{6,3,2}^{(4)}(\tau)+h_{6,5,2}^{(4)}(\tau) \\
& b_{3}(\tau)=h_{6,1,0}^{(4)}(\tau) \\
& b_{4}(\tau)=h_{6,1,3}^{(4)}(\tau)+h_{6,4,3}^{(4)}(\tau) .
\end{aligned}
$$

Alternatively, the elements $b_{i}(\tau)$ can be expressed in terms of the funtions $f_{n, p}(\tau)$ defined in eq. (3.25),

$$
\begin{aligned}
& b_{1}(\tau)=-\frac{91}{2880} f_{4,0}(\tau)+\frac{3}{80} f_{4,1}(\tau)-\frac{7}{864} f_{4,2}(\tau)+\frac{1}{2160} f_{4,3}(\tau)+\frac{7}{77760} f_{4,4}(\tau) \\
& b_{2}(\tau)=\frac{13}{720} f_{4,0}(\tau)-\frac{1}{180} f_{4,1}(\tau)+\frac{1}{216} f_{4,2}(\tau)-\frac{1}{180} f_{4,3}(\tau)-\frac{1}{19440} f_{4,4}(\tau) \\
& b_{3}(\tau)=-\frac{1}{80} f_{4,0}(\tau)-\frac{3}{20} f_{4,1}(\tau)+\frac{1}{72} f_{4,2}(\tau)-\frac{1}{540} f_{4,3}(\tau)+\frac{13}{19440} f_{4,4}(\tau) \\
& b_{4}(\tau)=\frac{7}{320} f_{4,0}(\tau)+\frac{1}{80} f_{4,1}(\tau)-\frac{7}{288} f_{4,2}(\tau)+\frac{1}{80} f_{4,3}(\tau)-\frac{91}{77760} f_{4,4}(\tau)
\end{aligned}
$$


Using these relations, we can express every iterated integral in eq. (4.11) in terms of the iterated integrals defined in eq. (4.16). We find

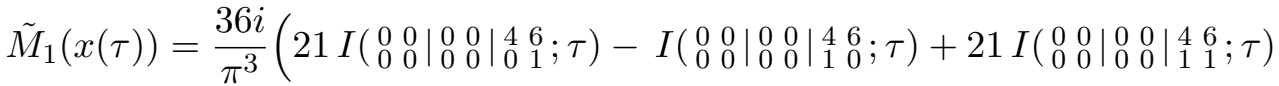

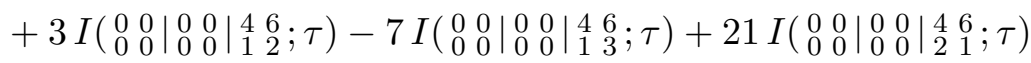

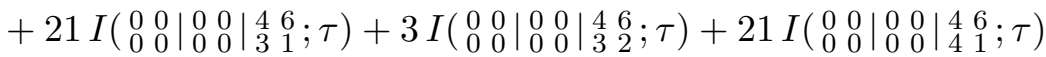

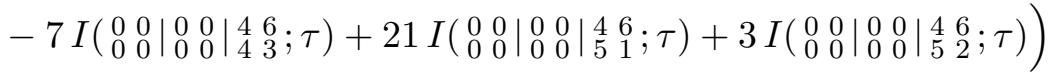

$$
\begin{aligned}
& -\frac{4 \zeta_{3}}{\pi^{2}}
\end{aligned}
$$

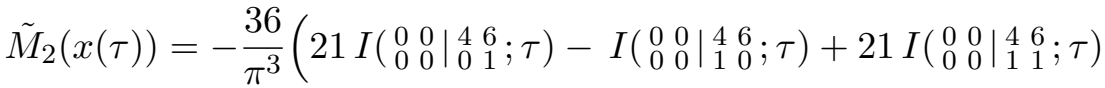

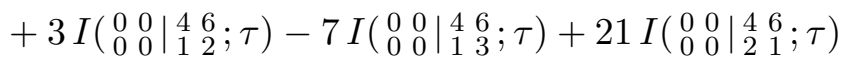

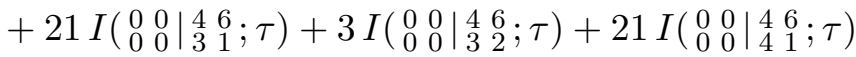

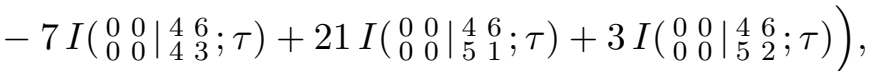

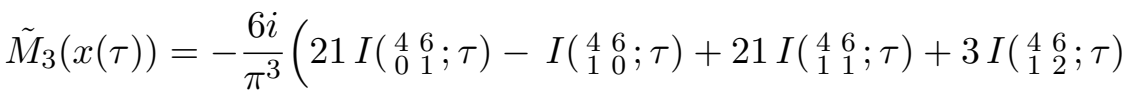

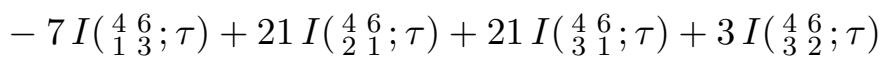

$$
\begin{aligned}
& \left.+21 I\left({ }_{4}^{4} \underset{1}{6} ; \tau\right)-7 I\left({ }_{4}^{4} \underset{3}{6} ; \tau\right)+21 I\left({ }_{5}^{4} \underset{1}{6} ; \tau\right)+3 I\left({ }_{5}^{4} \underset{2}{6} ; \tau\right)\right) \text {. }
\end{aligned}
$$

This result allows us to make the connection to eMPLs. In order to find a representation of the $\tilde{M}_{i}$ in terms of eMPLs, we write a suitable ansatz for them in terms of eMPLs, rewrite these eMPLs in terms of iterated integrals of Eisenstein series and then fix the coefficients in the ansatz with the results given in eq. (4.19). The ansatz we have chosen mirrors the observation that the $\tilde{M}_{i}$ are linear combinations of iterated integrals of modular forms of uniform weight and length, that all iterated integrals have leading zero entries and that only the last entry is a nontrivial modular form. Consequently, we chose elliptic multiple polylogarithms of the form

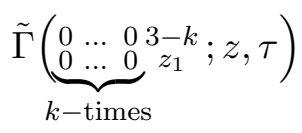

evaluated at rational points such that all solutions are related by adding or removing leading zero entries. More precisely, our ansatz consists of all antisymmetric combinations

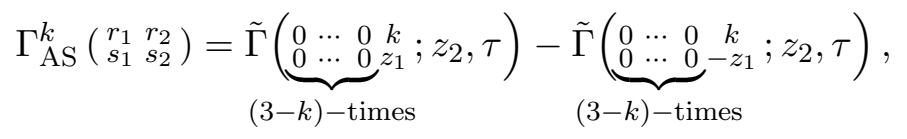

with $z_{i}=\frac{r_{i}}{6}+\frac{s_{i}}{6} \tau$ for $r_{i}, s_{i} \in\{0, \ldots, 5\}$ and for $1 \leq k \leq 3$.

We find that the solutions $\tilde{M}_{i}$ can indeed be expressed in terms of elliptic multiple polylogarithms and a possible representation is given by (for $k=1,2,3$ )

$$
\tilde{M}_{k}=C_{k} \tilde{m}_{k},
$$

where the prefactors are given by

$$
C_{1}=-12, \quad C_{2}=-\frac{6}{\pi}, \quad C_{3}=-\frac{1}{\pi^{2}},
$$


and the functions $m_{k}$ are defined as

$$
\begin{aligned}
& \tilde{m}_{k}=-\frac{13319}{96} \Gamma_{\mathrm{AS}}^{k}\left(\begin{array}{ll}
0 & 3 \\
1 & 0
\end{array}\right)+\frac{2679}{160} \Gamma_{\mathrm{AS}}^{k}\left(\begin{array}{ll}
0 & 5 \\
1 & 4
\end{array}\right)-\frac{77}{10} \Gamma_{\mathrm{AS}}^{k}\left(\begin{array}{ll}
0 & 3 \\
2 & 0
\end{array}\right) \\
& -\frac{2911}{15} \Gamma_{\mathrm{AS}}^{k}\left(\begin{array}{ll}
0 & 0 \\
3 & 2
\end{array}\right)+\frac{20261}{1440} \Gamma_{\mathrm{AS}}^{k}\left(\begin{array}{ll}
0 & 1 \\
3 & 4
\end{array}\right)+\frac{577}{60} \Gamma_{\mathrm{AS}}^{k}\left(\begin{array}{ll}
0 & 2 \\
3 & 5
\end{array}\right)-\frac{22841}{120} \Gamma_{\mathrm{AS}}^{k}\left(\begin{array}{ll}
0 & 4 \\
3 & 1
\end{array}\right) \\
& +\frac{1639}{180} \Gamma_{\mathrm{AS}}^{k}\left(\begin{array}{ll}
0 & 4 \\
3 & 5
\end{array}\right)-\frac{755827}{7200} \Gamma_{\mathrm{AS}}^{k}\left(\begin{array}{ll}
0 & 5 \\
3 & 0
\end{array}\right)-\frac{1371547}{2160} \Gamma_{\mathrm{AS}}^{k}\left(\begin{array}{ll}
0 & 5 \\
3 & 2
\end{array}\right)+\frac{969431}{720} \Gamma_{\mathrm{AS}}^{k}\left(\begin{array}{ll}
0 & 5 \\
3 & 3
\end{array}\right) \\
& -\frac{1011209}{2160} \Gamma_{\mathrm{AS}}^{k}\left(\begin{array}{ll}
0 & 5 \\
3 & 5
\end{array}\right)+\frac{77}{20} \Gamma_{\mathrm{AS}}^{k}\left(\begin{array}{ll}
0 & 3 \\
4 & 0
\end{array}\right)-\frac{70291}{480} \Gamma_{\mathrm{AS}}^{k}\left(\begin{array}{ll}
0 & 3 \\
5 & 0
\end{array}\right)+\frac{2679}{160} \Gamma_{\mathrm{AS}}^{k}\left(\begin{array}{ll}
0 & 5 \\
5 & 4
\end{array}\right) \\
& -\frac{10409}{90} \Gamma_{\mathrm{AS}}^{k}\left(\begin{array}{ll}
1 & 0 \\
0 & 3
\end{array}\right)+\frac{2197}{300} \Gamma_{\mathrm{AS}}^{k}\left(\begin{array}{ll}
1 & 5 \\
0 & 5
\end{array}\right)-\frac{893}{120} \Gamma_{\mathrm{AS}}^{k}\left(\begin{array}{ll}
1 & 0 \\
1 & 3
\end{array}\right)+\frac{665}{6} \Gamma_{\mathrm{AS}}^{k}\left(\begin{array}{ll}
1 & 3 \\
1 & 0
\end{array}\right) \\
& -\frac{57739}{288} \Gamma_{\mathrm{AS}}^{k}\left(\begin{array}{ll}
1 & 0 \\
2 & 1
\end{array}\right)+\frac{36031}{1440} \Gamma_{\mathrm{AS}}^{k}\left(\begin{array}{ll}
1 & 0 \\
2 & 5
\end{array}\right)-\frac{140}{3} \Gamma_{\mathrm{AS}}^{k}\left(\begin{array}{ll}
1 & 3 \\
2 & 0
\end{array}\right)+\frac{14}{5} \Gamma_{\mathrm{AS}}^{k}\left(\begin{array}{ll}
1 & 3 \\
2 & 3
\end{array}\right) \\
& +\frac{22867}{360} \Gamma_{\mathrm{AS}}^{k}\left(\begin{array}{ll}
1 & 3 \\
3 & 0
\end{array}\right)-\frac{2069}{40} \Gamma_{\mathrm{AS}}^{k}\left(\begin{array}{ll}
1 & 4 \\
3 & 3
\end{array}\right)-\frac{1427}{40} \Gamma_{\mathrm{AS}}^{k}\left(\begin{array}{ll}
1 & 0 \\
4 & 3
\end{array}\right)+\frac{847}{40} \Gamma_{\mathrm{AS}}^{k}\left(\begin{array}{ll}
1 & 0 \\
4 & 4
\end{array}\right) \\
& +\frac{7343}{60} \Gamma_{\mathrm{AS}}^{k}\left(\begin{array}{ll}
1 & 3 \\
4 & 0
\end{array}\right)-\frac{1579}{120} \Gamma_{\mathrm{AS}}^{k}\left(\begin{array}{ll}
1 & 0 \\
5 & 3
\end{array}\right)-\frac{55}{8} \Gamma_{\mathrm{AS}}^{k}\left(\begin{array}{ll}
1 & 0 \\
5 & 4
\end{array}\right)+\frac{6207}{40} \Gamma_{\mathrm{AS}}^{k}\left(\begin{array}{ll}
2 & 0 \\
0 & 3
\end{array}\right)
\end{aligned}
$$

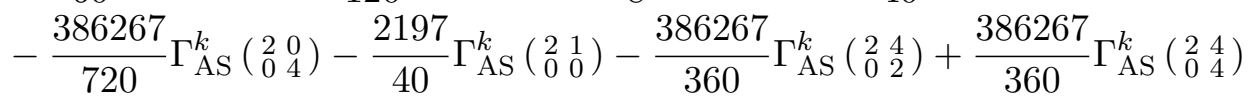

$$
\begin{aligned}
& -\frac{72913}{360} \Gamma_{\mathrm{AS}}^{k}\left(\begin{array}{ll}
2 & 3 \\
3 & 0
\end{array}\right)+\frac{1481}{20} \Gamma_{\mathrm{AS}}^{k}\left(\begin{array}{ll}
2 & 3 \\
3 & 3
\end{array}\right)+\frac{665}{12} \Gamma_{\mathrm{AS}}^{k}\left(\begin{array}{ll}
2 & 3 \\
4 & 0
\end{array}\right)+\frac{893}{60} \Gamma_{\mathrm{AS}}^{k}\left(\begin{array}{ll}
2 & 0 \\
5 & 3
\end{array}\right) \\
& -\frac{1367}{30} \Gamma_{\mathrm{AS}}^{k}\left(\begin{array}{ll}
3 & 0 \\
3 & 5
\end{array}\right)-\frac{188113}{10800} \Gamma_{\mathrm{AS}}^{k}\left(\begin{array}{ll}
3 & 2 \\
3 & 0
\end{array}\right)+\frac{105}{2} \Gamma_{\mathrm{AS}}^{k}\left(\begin{array}{ll}
3 & 3 \\
3 & 1
\end{array}\right)+\frac{263}{3} \Gamma_{\mathrm{AS}}^{k}\left(\begin{array}{ll}
3 & 3 \\
3 & 2
\end{array}\right) \\
& +\frac{1582769}{10800} \Gamma_{\mathrm{AS}}^{k}\left(\begin{array}{ll}
3 & 4 \\
3 & 0
\end{array}\right)-\frac{1555}{8} \Gamma_{\mathrm{AS}}^{k}\left(\begin{array}{ll}
3 & 5 \\
3 & 0
\end{array}\right)+\frac{77}{10} \Gamma_{\mathrm{AS}}^{k}\left(\begin{array}{ll}
3 & 3 \\
4 & 0
\end{array}\right)+\frac{203}{30} \Gamma_{\mathrm{AS}}^{k}\left(\begin{array}{ll}
3 & 0 \\
5 & 3
\end{array}\right) \\
& -\frac{21}{2} \Gamma_{\mathrm{AS}}^{k}\left(\begin{array}{ll}
3 & 3 \\
5 & 3
\end{array}\right)+\frac{14}{5} \Gamma_{\mathrm{AS}}^{k}\left(\begin{array}{ll}
3 & 3 \\
5 & 4
\end{array}\right)+\frac{8141}{120} \Gamma_{\mathrm{AS}}^{k}\left(\begin{array}{ll}
4 & 0 \\
1 & 3
\end{array}\right)+\frac{1271}{24} \Gamma_{\mathrm{AS}}^{k}\left(\begin{array}{ll}
4 & 3 \\
1 & 0
\end{array}\right) \\
& -\frac{1271}{24} \Gamma_{\mathrm{AS}}^{k}\left(\begin{array}{ll}
4 & 3 \\
1 & 3
\end{array}\right)-\frac{386267}{720} \Gamma_{\mathrm{AS}}^{k}\left(\begin{array}{ll}
4 & 0 \\
2 & 2
\end{array}\right)+\frac{386267}{720} \Gamma_{\mathrm{AS}}^{k}\left(\begin{array}{ll}
4 & 2 \\
2 & 0
\end{array}\right)+\frac{386267}{720} \Gamma_{\mathrm{AS}}^{k}\left(\begin{array}{ll}
4 & 2 \\
2 & 2
\end{array}\right) \\
& -\frac{665}{6} \Gamma_{\mathrm{AS}}^{k}\left(\begin{array}{ll}
4 & 3 \\
2 & 0
\end{array}\right)-\frac{386267}{720} \Gamma_{\mathrm{AS}}^{k}\left(\begin{array}{ll}
4 & 4 \\
2 & 0
\end{array}\right)-\frac{31277}{360} \Gamma_{\mathrm{AS}}^{k}\left(\begin{array}{ll}
4 & 3 \\
3 & 0
\end{array}\right)-\frac{147}{5} \Gamma_{\mathrm{AS}}^{k}\left(\begin{array}{ll}
4 & 3 \\
3 & 3
\end{array}\right) \\
& -\frac{386267}{360} \Gamma_{\mathrm{AS}}^{k}\left(\begin{array}{ll}
4 & 2 \\
4 & 0
\end{array}\right)+\frac{386267}{720} \Gamma_{\mathrm{AS}}^{k}\left(\begin{array}{ll}
4 & 4 \\
4 & 0
\end{array}\right)-\frac{253}{180} \Gamma_{\mathrm{AS}}^{k}\left(\begin{array}{ll}
4 & 0 \\
5 & 3
\end{array}\right)-\frac{1111}{10} \Gamma_{\mathrm{AS}}^{k}\left(\begin{array}{ll}
4 & 3 \\
5 & 0
\end{array}\right) \\
& -\frac{41}{12} \Gamma_{\mathrm{AS}}^{k}\left(\begin{array}{ll}
4 & 3 \\
5 & 2
\end{array}\right)+\frac{221}{60} \Gamma_{\mathrm{AS}}^{k}\left(\begin{array}{ll}
4 & 3 \\
5 & 3
\end{array}\right)+\frac{48}{5} \Gamma_{\mathrm{AS}}^{k}\left(\begin{array}{ll}
5 & 0 \\
1 & 1
\end{array}\right)+\frac{519}{40} \Gamma_{\mathrm{AS}}^{k}\left(\begin{array}{ll}
5 & 0 \\
1 & 3
\end{array}\right) \\
& -\frac{77}{2} \Gamma_{\mathrm{AS}}^{k}\left(\begin{array}{ll}
5 & 3 \\
1 & 3
\end{array}\right)+\frac{70}{6} \Gamma_{\mathrm{AS}}^{k}\left(\begin{array}{ll}
5 & 4 \\
1 & 0
\end{array}\right)+\frac{168}{5} \Gamma_{\mathrm{AS}}^{k}\left(\begin{array}{ll}
5 & 5 \\
1 & 0
\end{array}\right)-\frac{1045553}{720} \Gamma_{\mathrm{AS}}^{k}\left(\begin{array}{ll}
5 & 0 \\
2 & 2
\end{array}\right) \\
& +\frac{231407}{270} \Gamma_{\mathrm{AS}}^{k}\left(\begin{array}{ll}
5 & 0 \\
2 & 3
\end{array}\right)+\frac{1127}{60} \Gamma_{\mathrm{AS}}^{k}\left(\begin{array}{ll}
5 & 3 \\
2 & 0
\end{array}\right)+\frac{518}{5} \Gamma_{\mathrm{AS}}^{k}\left(\begin{array}{ll}
5 & 3 \\
2 & 3
\end{array}\right)+\frac{2069}{40} \Gamma_{\mathrm{AS}}^{k}\left(\begin{array}{ll}
5 & 0 \\
3 & 3
\end{array}\right) \\
& -\frac{9379}{360} \Gamma_{\mathrm{AS}}^{k}\left(\begin{array}{ll}
5 & 3 \\
3 & 0
\end{array}\right)-\frac{126}{5} \Gamma_{\mathrm{AS}}^{k}\left(\begin{array}{ll}
5 & 4 \\
3 & 0
\end{array}\right)+\frac{126}{5} \Gamma_{\mathrm{AS}}^{k}\left(\begin{array}{ll}
5 & 5 \\
3 & 0
\end{array}\right)-\frac{21637}{160} \Gamma_{\mathrm{AS}}^{k}\left(\begin{array}{ll}
5 & 0 \\
4 & 1
\end{array}\right) \\
& -\frac{1579}{160} \Gamma_{\mathrm{AS}}^{k}\left(\begin{array}{ll}
5 & 0 \\
4 & 5
\end{array}\right)+\frac{518}{5} \Gamma_{\mathrm{AS}}^{k}\left(\begin{array}{ll}
5 & 1 \\
4 & 1
\end{array}\right)+\frac{223}{10} \Gamma_{\mathrm{AS}}^{k}\left(\begin{array}{ll}
5 & 3 \\
4 & 0
\end{array}\right)+\frac{24}{5} \Gamma_{\mathrm{AS}}^{k}\left(\begin{array}{ll}
5 & 4 \\
4 & 0
\end{array}\right) \\
& +\frac{14}{5} \Gamma_{\mathrm{AS}}^{k}\left(\begin{array}{ll}
5 & 4 \\
4 & 5
\end{array}\right)-\frac{208783}{144} \Gamma_{\mathrm{AS}}^{k}\left(\begin{array}{ll}
5 & 0 \\
5 & 1
\end{array}\right)+\frac{1078601}{2160} \Gamma_{\mathrm{AS}}^{k}\left(\begin{array}{ll}
5 & 0 \\
5 & 3
\end{array}\right)-\frac{141}{10} \Gamma_{\mathrm{AS}}^{k}\left(\begin{array}{ll}
5 & 0 \\
5 & 5
\end{array}\right) \\
& -\frac{37841}{2700} \Gamma_{\mathrm{AS}}^{k}\left(\begin{array}{ll}
5 & 2 \\
5 & 0
\end{array}\right)+\frac{321817}{2700} \Gamma_{\mathrm{AS}}^{k}\left(\begin{array}{ll}
5 & 3 \\
5 & 0
\end{array}\right)-\frac{136121}{2700} \Gamma_{\mathrm{AS}}^{k}\left(\begin{array}{ll}
5 & 4 \\
5 & 0
\end{array}\right)+\frac{12277}{300} \Gamma_{\mathrm{AS}}^{k}\left(\begin{array}{ll}
5 & 5 \\
5 & 0
\end{array}\right) \text {. }
\end{aligned}
$$


We note here that the boundary constant proportional to $\zeta_{3}$, appearing in eq. (4.19) does not appear explicitly in this representation, as for $k=1$ the term proportional to $\zeta_{3}$ is contained in the combination of eMPLs.

\section{Conclusion and outlook}

In this paper we have presented for the first time fully analytic results for all master integrals of the equal-mass three-loop banana graph. Our results are characterised by remarkable simplicity, and they only involve the same class of functions that shows up also in the two-loop equal-mass sunrise graph, namely iterated integrals for modular forms for $\Gamma_{1}(6)$ and elliptic polylogarithms evaluated at rational points.

Our paper is also the first time that a family of Feynman integral whose associated Picard-Fuchs operator is irreducible of order three has been evaluated analytically in terms of a well-established class of transcendental functions. This result may have important implications for tackling phenomenologically relevant three-loop processes involving massive virtual particles. In particular, the banana graph is the simplest subtopology that appears in the computation of the three-loop corrections to Higgs production via gluon fusion where the dependence on the top-quark mass is kept. While these corrections are known numerically [80], no analytic solution is known. Correspondingly, the full analytic result will necessarily involve integrals over the banana graphs. Our results in terms of iterated integrals of modular forms are well suited to perform these integrals. Most likely, however, also higher orders in the $\epsilon$-expansion of the banana graph would be required and we expect that the techniques presented in this paper can be extended to this case as well. This is left for future work.

\section{A Boundary condition for the banana graph}

In this section we discuss how to obtain the leading asymptotic expansion of the master integrals for the banana integrals in eq. (4.5). Asymptotic expansions for Feynman integrals are a well studied topic in the context of the method of expansion-by-regions [81, 82]. Here we will employ a particular method that relies on Mellin-Barnes integral transformations to obtain the asymptotic expansion of the banana Feynman integrals around the point $x=0$.

We start by Feynman parametrising the integral. The Symanzik polynomials relevant for the three master integrals in eq. (2.4) are

$$
\begin{aligned}
& \mathcal{U}=x_{1} x_{2} x_{3}+x_{1} x_{4} x_{3}+x_{2} x_{4} x_{3}+x_{1} x_{2} x_{4} \text { and } \\
& \mathcal{F}=s x_{1} x_{2} x_{3} x_{4}+m^{2}\left(x_{1}+x_{2}+x_{3}+x_{4}\right) \mathcal{U},
\end{aligned}
$$

so that we can write the Feynman parametric representation of the first master integral as

$$
\begin{aligned}
\mathcal{I}_{1} & =(1+2 \epsilon)(1+3 \epsilon) I_{1,1,1,1,0,0,0,0,0} \\
& =\frac{(1+2 \epsilon)(1+3 \epsilon)}{\Gamma(1+\epsilon)^{3}} \int d x_{1} d x_{2} d x_{3} d x_{4} \delta\left(1-x_{4}\right) \mathcal{U}^{4 \epsilon} \mathcal{F}^{-1-3 \epsilon},
\end{aligned}
$$


where we have chosen the argument of the $\delta$ function in a way that is advantageous for the rest of the calculation.

We can use a useful trick to simplify the integral drastically, at the cost of introducing an additional integration: we introduce a Mellin-Barnes parameter by using the identity,

$$
\frac{1}{(A+B)^{\lambda}}=\int_{\mathcal{C}-i \infty}^{\mathcal{C}+i \infty} \frac{d \xi}{2 \pi i} A^{\xi} B^{-\xi-\lambda} \frac{\Gamma(-\xi) \Gamma(\xi+\lambda)}{\Gamma(\lambda)}
$$

where the contour of integration runs parallel to the imaginary axis and intersects the real axis at a point $\mathcal{C}$ that is chosen such that the contour separates the left poles of the integrand (due to $\Gamma(\xi+\lambda)$ ) from the right poles (due to $\Gamma(-\xi)$ ). We can use this identity to separate the two terms in the $\mathcal{F}$ polynomial and write the integral as

$$
\begin{aligned}
\mathcal{I}_{1}= & \int \frac{d \xi_{1}}{2 \pi i} \frac{\Gamma\left(-\xi_{1}\right) \Gamma\left(1+3 \epsilon+\xi_{1}\right)}{\Gamma(1+\epsilon)^{3}}(-x / 4)^{-\xi_{1}} \\
& \times \int d x_{1} d x_{2} d x_{3} d x_{4} \delta\left(1-x_{4}\right)\left(x_{1} x_{2} x_{3} x_{4}\right)^{\xi_{1}}\left(x_{1}+x_{2}+x_{3}+x_{4}\right)^{-1-3 \epsilon-\xi_{1}} \mathcal{U}^{-1+\epsilon-\xi_{1}}
\end{aligned}
$$

This transformation renders the integral effectively massless and we can proceed to integrate out the Feynman parameters $x_{i}$ one at a time. In doing so we encounter two integrals of the form

$$
\int_{0}^{\infty} d x x^{\alpha}(A+B x)^{\beta}(C+D x)^{\gamma}
$$

Ordinarily, such an integral can be evaluated in terms of hypergeometric functions. However, in this case it is advantageous to instead apply the Mellin-Barns trick from eq. (A.3) once more, in order to split one of the two linear terms into monomial factors, which will allow us to perform the integral in terms of $\Gamma$ functions as

$$
\int_{0}^{\infty} d x x^{\alpha}(A+B x)^{\beta}=A^{1+\alpha+\beta} B^{-1-\alpha} \frac{\Gamma(1+\alpha) \Gamma(-1-\alpha-\beta)}{\Gamma(-\beta)} .
$$

After integrating out the Feynman parameters in this fashion, we find the following MellinBarnes representation of the integral,

$$
\begin{aligned}
\mathcal{I}_{1}= & (1+2 \epsilon)(1+3 \epsilon) \int \frac{d \xi_{1} d \xi_{2} d \xi_{3}}{(2 \pi i)^{3}}(-x / 4)^{-\xi_{1}} \Gamma\left(-\xi_{1}\right) \Gamma\left(-\xi_{2}\right) \Gamma\left(-\xi_{3}\right) \\
& \times \Gamma\left(\epsilon-\xi_{2}\right) \Gamma\left(\epsilon-\xi_{3}\right) \frac{\Gamma\left(1+\xi_{123}\right)^{2} \Gamma\left(1-\epsilon+\xi_{123}\right) \Gamma\left(1+\epsilon+\xi_{123}\right)}{\Gamma(1+\epsilon)^{3} \Gamma\left(2+2 \xi_{123}\right) \Gamma\left(1-\epsilon+\xi_{1}\right)},
\end{aligned}
$$

where we have defined the abbreviation $\xi_{123}=\xi_{1}+\xi_{2}+\xi_{3}$. In the above integral, the contour of integration is defined implicitly through the requirement that it separates the left and right poles of the $\Gamma$ functions. An explicit representation of the contour can be obtained in an algorithmic fashion as implemented for example in the Mathematica packages MB [83] and MBresolve [84]. The explicit form of the contour is useful when using Cauchy's theorem to perform the remaining integrations, by closing the contour and summing residues. However, we will see that this is actually not necessary in this case.

First of all, so far we have not performed any asymptotic expansion and eq. (A.7) is a Mellin-Barnes representation of the entire integral, but we only care about the integral in the limit $x \rightarrow 0$. 
The Mellin-Barnes representation allows us to take the asymptotic limit in a straightforward fashion: inspecting the integrand of the Mellin-Barnes representation we see that for generic values of the $\xi_{i}$ the integrand vanishes when we take $x \rightarrow 0$. To obtain the Laurent expansion around vanishing $x$, we therefore need to take the leading residues, starting from $\xi_{1}=1$. The surviving residues can be determined algorithmically for example using the package MBasymptotics [85]. Solving the constraints for our integral in eq. (A.7) we find that the only terms contributing in the limit $x \rightarrow 0$ are codimension three residues so that no integrations remain. We have,

$$
\begin{aligned}
\frac{\lim _{x \rightarrow 0} \mathcal{I}_{1}}{(1+2 \epsilon)(1+3 \epsilon)}= & -\frac{x}{\epsilon^{3}}-\frac{12 \Gamma(1-\epsilon)^{2}}{\epsilon^{3} \Gamma(1-2 \epsilon)}(-x / 4)^{1+\epsilon}+\frac{12 \Gamma(1-\epsilon)^{3} \Gamma(1+2 \epsilon)}{\epsilon^{3} \Gamma(1-3 \epsilon) \Gamma(1+\epsilon)^{2}}(-x / 4)^{1+2 \epsilon} \\
& -\frac{4 \Gamma(1-\epsilon)^{4} \Gamma(1+3 \epsilon)}{\epsilon^{3} \Gamma(1-4 \epsilon) \Gamma(1+\epsilon)^{3}}(-x / 4)^{1+3 \epsilon} \\
= & x\left[\log (-x / 4)^{3}-4 \zeta_{3}\right] \\
& +\epsilon x\left[-6 \zeta_{4}-20 \zeta_{3}-30 \zeta_{3} \log (-x / 4)-3 \zeta_{2} \log (-x / 4)^{2}\right. \\
& \left.+5 \log (-x / 4)^{3}+\frac{3}{2} \log (-x / 4)^{4}\right]+\mathcal{O}\left(\epsilon^{2}\right)
\end{aligned}
$$

The other two master integrals can be computed completely analogously, the only difference are shifted exponents of the Symanzik polynomials, and we can obtain the asymptotic limit for the second master integral as

$$
\begin{aligned}
\frac{\lim _{x \rightarrow 0} \mathcal{I}_{2}}{(1+2 \epsilon)}= & \frac{3}{4 \epsilon^{2}} x+\frac{6 \Gamma(1-\epsilon)^{2}}{\epsilon^{2} \Gamma(1-2 \epsilon)}(-x / 4)^{1+\epsilon}-\frac{3 \Gamma(1-\epsilon)^{3} \Gamma(1+2 \epsilon)}{\epsilon^{2} \Gamma(1-3 \epsilon) \Gamma(1+\epsilon)^{2}}(-x / 4)^{1+2 \epsilon} \\
=\left[\frac{3}{4} x \log (-x / 4)^{2}\right]+\epsilon x & {\left[\frac{9}{2} \zeta_{3}-\frac{3}{2} \zeta_{2} \log (-x / 4)+\frac{3}{2} x \log (-x / 4)^{2}\right.} \\
& \left.+\frac{3}{4} \log (-x / 4)^{3}\right]+\mathcal{O}\left(\epsilon^{2}\right),
\end{aligned}
$$

and similarly for the third master integral,

$$
\begin{aligned}
\lim _{x \rightarrow 0} \mathcal{I}_{3} & =-\frac{1}{2 \epsilon} x-\frac{2 \Gamma(1-\epsilon)^{2}}{\epsilon \Gamma(1-2 \epsilon)}(-x / 4)^{1+\epsilon} \\
& =\left[\frac{1}{2} x \log (-x / 4)\right]+\epsilon x\left[-\frac{1}{2} \zeta_{2}+\frac{1}{4} \log (-x / 4)^{2}\right]+\mathcal{O}\left(\epsilon^{2}\right) .
\end{aligned}
$$

\section{B Decomposing a matrix into a semi-simple and a unipotent part}

In this appendix we show how to decompose an invertible matrix $\Omega$ (with certain additional conditions, see below) into a product of a lower and upper-triangular matrix. From this we can infer the decomposition of the period matrix of the banana graph into a semi-simple and a unipotent matrix, see eq. (4.7). A unipotent matrix is a matrix whose difference to the unit matrix is nilpotent. Good examples are upper triangular matrices with only ones on the diagonal. A semi-simple matrix, on the other hand, is a matrix which is similar 
to a direct sum of simple matrices. Over an algebraically closed field (e.g., the complex numbers), semi-simple matrices are just the diagonalisable matrices.

Let us define the matrix

$$
\Omega=\left(\Omega_{i j}\right)_{1 \leq i, j \leq n},
$$

which we assume to be invertible. In the following we are going to show that the matrix can be decomposed into the upper-triangular matrix $U$ and a lower-triangular matrix $S$ such that

$$
\Omega=S U
$$

In order to do so, consider the principle minors of $\Omega$,

$$
M_{k}=\operatorname{det}\left(\Omega_{i j}\right)_{1 \leq i, j \leq k}=\operatorname{det} \Omega^{(k)}, \quad M_{0} \equiv 1 .
$$

Furthermore, let us define auxiliary sets of matrices:

$$
O_{k \ell}=\operatorname{det}\left(\begin{array}{cccc}
\Omega_{11} & \cdots & \Omega_{1 k-1} & \Omega_{1 \ell} \\
\vdots & & \vdots & \vdots \\
\Omega_{k 1} & \cdots & \Omega_{k k-1} & \Omega_{k \ell}
\end{array}\right), \quad C_{k \ell}=\operatorname{det}\left(\begin{array}{ccc}
\Omega_{11} & \cdots & \Omega_{1 k} \\
\vdots & & \vdots \\
\Omega_{k-11} & \cdots & \Omega_{k-1 k} \\
\Omega_{\ell 1} & \cdots & \Omega_{\ell k}
\end{array}\right) .
$$

Using those auxiliary objects, define the matrices:

$$
S_{i j}=\frac{C_{j i}}{M_{j-1}} \text { and } U_{i j}=\frac{O_{i j}}{M_{i}} .
$$

Note that for the previous equation to make sense, we need to require that all principle minors of $\Omega$ be non-zero.

Writing out the product of $S$ and $U$, one finds

$$
\begin{aligned}
\sum_{k=1}^{n} S_{i k} U_{k j} & =\sum_{k=1}^{n} C_{k i} O_{k j} \\
& =\sum_{k=1}^{n} \frac{1}{M_{k} M_{k-1}} \operatorname{det}\left(\begin{array}{cc}
\Omega^{(k-1)} & \Omega_{* k} \\
\Omega_{i *} & \Omega_{i k}
\end{array}\right) \operatorname{det}\left(\begin{array}{cc}
\Omega^{(k-1)} & \Omega_{* j} \\
\Omega_{k *} & \Omega_{k j}
\end{array}\right) \\
& =\sum_{k=1}^{n} \frac{1}{\operatorname{det} \Omega^{(k)} \operatorname{det} \Omega^{(k-1)}} \operatorname{det}\left(\begin{array}{cc}
\Omega^{(k-1)} & \Omega_{* k} \\
\Omega_{i *} & \Omega_{i k}
\end{array}\right) \operatorname{det}\left(\begin{array}{cc}
\Omega^{(k-1)} & \Omega_{* j} \\
\Omega_{k *} & \Omega_{k j}
\end{array}\right) \\
& =\Omega_{i j} .
\end{aligned}
$$

The manipulations in the above equation for generic matrices are algebraically rather involved. We have therefore limited ourselves to testing explicitly the correctness of this formula for matrices up to $n=10$.

Next, we note that the matrix $U$ has the following shape:

$$
\left(\begin{array}{lll}
1 & & * \\
& \ddots & \\
0 & & 1
\end{array}\right)
$$


This immediately implies that $U$ is unipotent. Indeed, considering $i>j$, one finds

$$
U_{i j}=O_{i j}=\frac{1}{M_{i}} \operatorname{det}\left(\begin{array}{cccc}
\Omega_{11} & \cdots & \Omega_{1 i-1} & \Omega_{1 j} \\
\vdots & & \vdots & \vdots \\
\Omega_{i 1} & \cdots & \Omega_{i i-1} & \Omega_{i j}
\end{array}\right)=0
$$

because there are two identical columns. For the diagonal elements one finds

$$
U_{i i}=O_{i i}=\frac{1}{M_{i}} M_{i}=1 .
$$

One can show along the same lines that all elements $S_{i j}$ for $i<j$ vanish, and so $S$ is lower-triangular.

The previous considerations do not yet allow us to conclude that $S$ is semi-simple, because not every lower-triangular matrix is diagonalisable. We can, however, easily check that the matrix $S$ obtained in this way is diagonalisable on a case by case basis. Indeed, a sufficient criterion for a triangular matrix to be diagonalisable is that all its diagonal elements are distinct (because in that case the matrix has a maximal number of distinct eigenvalues). In particular, we can then easily check that this construction leads to a semisimple matrix $S$ in the case of the banana graph where $\Omega=\mathcal{W}(x)$, with $\mathcal{W}$ is defined in eq. (2.15). Indeed, we immediately see that in that case $S$ has three distinct eigenvalues for generic values of $x$. Therefore, $S$ is diagonalisable for generic $x$, and hence semi-simple. We have thus obtained the desired decomposition into a semi-simple and a unipotent matrix.

Open Access. This article is distributed under the terms of the Creative Commons Attribution License (CC-BY 4.0), which permits any use, distribution and reproduction in any medium, provided the original author(s) and source are credited.

\section{References}

[1] E.E. Kummer, Über die Transcendenten, welche aus wiederholten Integrationen rationaler Formeln entstehen (in German), J. Reine Ang. Math. 21 (1840) 74 [Ibid. 21 (1840) 193] [Ibid. 21 (1840) 328].

[2] N. Nielsen, Der Eulersche Dilogarithmus und seine Verallgemeinerungen (in German), Nova Acta Leopoldina (Halle) 90 (1909) 123.

[3] A.B. Goncharov, Multiple polylogarithms, cyclotomy and modular complexes, Math. Res. Lett. 5 (1998) 497 [arXiv:1105.2076] [InSPIRE].

[4] D.J. Broadhurst, The master two loop diagram with masses, Z. Phys. C 47 (1990) 115 [INSPIRE].

[5] S. Bauberger, F.A. Berends, M. Böhm and M. Buza, Analytical and numerical methods for massive two loop selfenergy diagrams, Nucl. Phys. B 434 (1995) 383 [hep-ph/9409388] [INSPIRE].

[6] S. Bauberger and M. Böhm, Simple one-dimensional integral representations for two loop selfenergies: the master diagram, Nucl. Phys. B 445 (1995) 25 [hep-ph/9501201] [INSPIRE].

[7] S. Laporta and E. Remiddi, Analytic treatment of the two loop equal mass sunrise graph, Nucl. Phys. B 704 (2005) 349 [hep-ph/0406160] [INSPIRE]. 
[8] B.A. Kniehl, A.V. Kotikov, A. Onishchenko and O. Veretin, Two-loop sunset diagrams with three massive lines, Nucl. Phys. B 738 (2006) 306 [hep-ph/0510235] [INSPIRE].

[9] U. Aglietti, R. Bonciani, L. Grassi and E. Remiddi, The two loop crossed ladder vertex diagram with two massive exchanges, Nucl. Phys. B 789 (2008) 45 [arXiv:0705.2616] [INSPIRE].

[10] M. Czakon and A. Mitov, Inclusive heavy flavor hadroproduction in NLO QCD: the exact analytic result, Nucl. Phys. B $\mathbf{8 2 4}$ (2010) 111 [arXiv:0811.4119] [INSPIRE].

[11] F. Brown and O. Schnetz, A K3 in $\phi^{4}$, Duke Math. J. 161 (2012) 1817 [arXiv:1006.4064] [INSPIRE].

[12] S. Müller-Stach, S. Weinzierl and R. Zayadeh, A second-order differential equation for the two-loop sunrise graph with arbitrary masses, Commun. Num. Theor. Phys. 6 (2012) 203 [arXiv: 1112.4360] [INSPIRE].

[13] S. Caron-Huot and K.J. Larsen, Uniqueness of two-loop master contours, JHEP 10 (2012) 026 [arXiv: 1205.0801] [INSPIRE].

[14] R. Huang and Y. Zhang, On genera of curves from high-loop generalized unitarity cuts, JHEP 04 (2013) 080 [arXiv: 1302.1023] [INSPIRE].

[15] F. Brown and O. Schnetz, Modular forms in quantum field theory, Commun. Num. Theor Phys. 07 (2013) 293 [arXiv:1304.5342] [InSPIRE].

[16] D. Nandan, M.F. Paulos, M. Spradlin and A. Volovich, Star integrals, convolutions and simplices, JHEP 05 (2013) 105 [arXiv: 1301.2500] [INSPIRE].

[17] S. Bloch and P. Vanhove, The elliptic dilogarithm for the sunset graph, J. Number Theor. 148 (2015) 328 [arXiv:1309.5865] [INSPIRE].

[18] L. Adams, C. Bogner and S. Weinzierl, The two-loop sunrise graph with arbitrary masses, J. Math. Phys. 54 (2013) 052303 [arXiv:1302.7004] [INSPIRE].

[19] L. Adams, C. Bogner and S. Weinzierl, The two-loop sunrise graph in two space-time dimensions with arbitrary masses in terms of elliptic dilogarithms, J. Math. Phys. 55 (2014) 102301 [arXiv:1405.5640] [INSPIRE].

[20] L. Adams, C. Bogner and S. Weinzierl, The two-loop sunrise integral around four space-time dimensions and generalisations of the Clausen and Glaisher functions towards the elliptic case, J. Math. Phys. 56 (2015) 072303 [arXiv:1504.03255] [INSPIRE].

[21] L. Adams, C. Bogner and S. Weinzierl, The iterated structure of the all-order result for the two-loop sunrise integral, J. Math. Phys. 57 (2016) 032304 [arXiv:1512.05630] [INSPIRE].

[22] E. Remiddi and L. Tancredi, Differential equations and dispersion relations for Feynman amplitudes. The two-loop massive sunrise and the kite integral, Nucl. Phys. B 907 (2016) 400 [arXiv: 1602.01481] [INSPIRE].

[23] A. Primo and L. Tancredi, On the maximal cut of Feynman integrals and the solution of their differential equations, Nucl. Phys. B 916 (2017) 94 [arXiv:1610.08397] [InSPIRE].

[24] R. Bonciani, V. Del Duca, H. Frellesvig, J.M. Henn, F. Moriello and V.A. Smirnov, Two-loop planar master integrals for Higgs $\rightarrow 3$ partons with full heavy-quark mass dependence, JHEP 12 (2016) 096 [arXiv:1609.06685] [INSPIRE].

[25] L. Adams, C. Bogner, A. Schweitzer and S. Weinzierl, The kite integral to all orders in terms of elliptic polylogarithms, J. Math. Phys. 57 (2016) 122302 [arXiv:1607.01571] [InSPIRE]. 
[26] G. Passarino, Elliptic polylogarithms and basic hypergeometric functions, Eur. Phys. J. C 77 (2017) 77 [arXiv:1610.06207] [INSPIRE].

[27] A. von Manteuffel and L. Tancredi, A non-planar two-loop three-point function beyond multiple polylogarithms, JHEP 06 (2017) 127 [arXiv: 1701.05905] [INSPIRE].

[28] J. Ablinger et al., Iterated elliptic and hypergeometric integrals for Feynman diagrams, J. Math. Phys. 59 (2018) 062305 [arXiv:1706.01299] [INSPIRE].

[29] L.-B. Chen, Y. Liang and C.-F. Qiao, $N N L O Q C D$ corrections to $\gamma+\eta_{c}\left(\eta_{b}\right)$ exclusive production in electron-positron collision, JHEP 01 (2018) 091 [arXiv:1710.07865] [INSPIRE].

[30] M. Hidding and F. Moriello, All orders structure and efficient computation of linearly reducible elliptic Feynman integrals, JHEP 01 (2019) 169 [arXiv:1712.04441] [INSPIRE].

[31] C. Bogner, A. Schweitzer and S. Weinzierl, Analytic continuation and numerical evaluation of the kite integral and the equal mass sunrise integral, Nucl. Phys. B 922 (2017) 528 [arXiv: 1705.08952] [INSPIRE].

[32] J.L. Bourjaily, A.J. McLeod, M. Spradlin, M. von Hippel and M. Wilhelm, Elliptic double-box integrals: massless scattering amplitudes beyond polylogarithms, Phys. Rev. Lett. 120 (2018) 121603 [arXiv:1712.02785] [INSPIRE].

[33] J. Broedel, C. Duhr, F. Dulat and L. Tancredi, Elliptic polylogarithms and iterated integrals on elliptic curves II: an application to the sunrise integral, Phys. Rev. D 97 (2018) 116009 [arXiv: 1712.07095] [INSPIRE].

[34] S. Laporta, High-precision calculation of the 4-loop contribution to the electron $g-2$ in QED, Phys. Lett. B 772 (2017) 232 [arXiv:1704.06996] [INSPIRE].

[35] J. Broedel, C. Duhr, F. Dulat, B. Penante and L. Tancredi, Elliptic symbol calculus: from elliptic polylogarithms to iterated integrals of Eisenstein series, JHEP 08 (2018) 014 [arXiv: 1803.10256] [INSPIRE].

[36] J. Broedel, C. Duhr, F. Dulat, B. Penante and L. Tancredi, Elliptic Feynman integrals and pure functions, JHEP 01 (2019) 023 [arXiv:1809.10698] [INSPIRE].

[37] L. Adams, E. Chaubey and S. Weinzierl, Planar double box integral for top pair production with a closed top loop to all orders in the dimensional regularization parameter, Phys. Rev. Lett. 121 (2018) 142001 [arXiv:1804.11144] [INSPIRE].

[38] L. Adams, E. Chaubey and S. Weinzierl, Analytic results for the planar double box integral relevant to top-pair production with a closed top loop, JHEP 10 (2018) 206 [arXiv: 1806.04981] [INSPIRE].

[39] J. Broedel, C. Duhr, F. Dulat, B. Penante and L. Tancredi, Elliptic polylogarithms and Feynman parameter integrals, JHEP 05 (2019) 120 [arXiv:1902.09971] [INSPIRE].

[40] C. Bogner, S. Müller-Stach and S. Weinzierl, The unequal mass sunrise integral expressed through iterated integrals on $\overline{\mathcal{M}}_{1,3}$, arXiv:1907.01251 [INSPIRE].

[41] F. Brown and A. Levin, Multiple elliptic polylogarithms, arXiv:1110.6917.

[42] E. Remiddi and L. Tancredi, An elliptic generalization of multiple polylogarithms, Nucl. Phys. B 925 (2017) 212 [arXiv:1709.03622] [INSPIRE]. 
[43] J. Broedel, C. Duhr, F. Dulat and L. Tancredi, Elliptic polylogarithms and iterated integrals on elliptic curves. Part I: general formalism, JHEP 05 (2018) 093 [arXiv:1712.07089] [INSPIRE].

[44] Y.I. Manin, Iterated integrals of modular forms and noncommutative modular symbols, in Algebraic geometry and number theory, Progr. Math. 253, Birkhäuser, Boston, MA, U.S.A. (2006), pg. 565 [math.NT/0502576].

[45] F. Brown, Multiple modular values and the relative completion of the fundamental group of $\mathcal{M}_{1,1}$, arXiv: 1407.5167 .

[46] L. Adams and S. Weinzierl, Feynman integrals and iterated integrals of modular forms, Commun. Num. Theor. Phys. 12 (2018) 193 [arXiv:1704.08895] [INSPIRE].

[47] J. Broedel, C.R. Mafra, N. Matthes and O. Schlotterer, Elliptic multiple zeta values and one-loop superstring amplitudes, JHEP 07 (2015) 112 [arXiv:1412.5535] [INSPIRE].

[48] J. Broedel, N. Matthes and O. Schlotterer, Relations between elliptic multiple zeta values and a special derivation algebra, J. Phys. A 49 (2016) 155203 [arXiv:1507.02254] [INSPIRE].

[49] J. Broedel, N. Matthes, G. Richter and O. Schlotterer, Twisted elliptic multiple zeta values and non-planar one-loop open-string amplitudes, J. Phys. A 51 (2018) 285401 [arXiv: 1704.03449] [INSPIRE].

[50] J. Broedel, O. Schlotterer and F. Zerbini, From elliptic multiple zeta values to modular graph functions: open and closed strings at one loop, JHEP 01 (2019) 155 [arXiv:1803.00527] [INSPIRE].

[51] S. Bloch, M. Kerr and P. Vanhove, A Feynman integral via higher normal functions, Compos. Math. 151 (2015) 2329 [arXiv:1406.2664] [INSPIRE].

[52] S. Bloch, M. Kerr and P. Vanhove, Local mirror symmetry and the sunset Feynman integral, Adv. Theor. Math. Phys. 21 (2017) 1373 [arXiv:1601.08181] [InSPIRE].

[53] D. Broadhurst, Feynman integrals, L-series and Kloosterman moments, Commun. Num. Theor. Phys. 10 (2016) 527 [arXiv:1604.03057] [InSPIRE].

[54] J.L. Bourjaily, A.J. McLeod, M. von Hippel and M. Wilhelm, Bounded collection of Feynman integral Calabi-Yau geometries, Phys. Rev. Lett. 122 (2019) 031601 [arXiv:1810.07689] [INSPIRE].

[55] J.L. Bourjaily, Y.-H. He, A.J. Mcleod, M. Von Hippel and M. Wilhelm, Traintracks through Calabi-Yau manifolds: scattering amplitudes beyond elliptic polylogarithms, Phys. Rev. Lett. 121 (2018) 071603 [arXiv:1805.09326] [INSPIRE].

[56] H.A. Verrill, Root lattices and pencils of varieties, J. Math. Kyoto Univ. 36 (1996) 423.

[57] A. Primo and L. Tancredi, Maximal cuts and differential equations for Feynman integrals. An application to the three-loop massive banana graph, Nucl. Phys. B 921 (2017) 316 [arXiv: 1704.05465] [INSPIRE].

[58] P. Mastrolia and E. Remiddi, The analytic value of a three loop sunrise graph in a particular kinematical configuration, Nucl. Phys. B 657 (2003) 397 [hep-ph/0211451] [INSPIRE].

[59] K.G. Chetyrkin and F.V. Tkachov, Integration by parts: the algorithm to calculate $\beta$-functions in 4 loops, Nucl. Phys. B 192 (1981) 159 [INSPIRE].

[60] F.V. Tkachov, A theorem on analytical calculability of four loop renormalization group functions, Phys. Lett. B 100 (1981) 65 [INSPIRE]. 
[61] O.V. Tarasov, Connection between Feynman integrals having different values of the space-time dimension, Phys. Rev. D 54 (1996) 6479 [hep-th/9606018] [INSPIRE].

[62] V.A. Smirnov and O.L. Veretin, Analytical results for dimensionally regularized massless on-shell double boxes with arbitrary indices and numerators, Nucl. Phys. B 566 (2000) 469 [hep-ph/9907385] [INSPIRE].

[63] C. Anastasiou, E.W.N. Glover and C. Oleari, The two-loop scalar and tensor pentabox graph with light-like legs, Nucl. Phys. B 575 (2000) 416 [Erratum ibid. B 585 (2000) 763] [hep-ph/9912251] [INSPIRE].

[64] C. Anastasiou, T. Gehrmann, C. Oleari, E. Remiddi and J.B. Tausk, The tensor reduction and master integrals of the two loop massless crossed box with lightlike legs, Nucl. Phys. B 580 (2000) 577 [hep-ph/0003261] [INSPIRE].

[65] C. Anastasiou, J.B. Tausk and M.E. Tejeda-Yeomans, The on-shell massless planar double box diagram with an irreducible numerator, Nucl. Phys. Proc. Suppl. 89 (2000) 262 [hep-ph/0005328] [INSPIRE].

[66] R.N. Lee, Space-time dimensionality D as complex variable: calculating loop integrals using dimensional recurrence relation and analytical properties with respect to $D$, Nucl. Phys. B 830 (2010) 474 [arXiv:0911.0252] [InSPIRE].

[67] A.V. Kotikov, Differential equations method: new technique for massive Feynman diagrams calculation, Phys. Lett. B 254 (1991) 158 [INSPIRE].

[68] A.V. Kotikov, Differential equations method: the calculation of vertex type Feynman diagrams, Phys. Lett. B 259 (1991) 314 [INSPIRE].

[69] A.V. Kotikov, Differential equation method: the calculation of $N$ point Feynman diagrams, Phys. Lett. B 267 (1991) 123 [Erratum ibid. B 295 (1992) 409] [InSPIRE].

[70] T. Gehrmann and E. Remiddi, Differential equations for two loop four point functions, Nucl. Phys. B 580 (2000) 485 [hep-ph/9912329] [InSPIRE].

[71] T. Gehrmann and E. Remiddi, Two loop master integrals for $\gamma^{*} \rightarrow 3$ jets: the planar topologies, Nucl. Phys. B 601 (2001) 248 [hep-ph/0008287] [InSPIRE].

[72] H. Frellesvig and C.G. Papadopoulos, Cuts of Feynman integrals in Baikov representation, JHEP 04 (2017) 083 [arXiv:1701.07356] [INSPIRE].

[73] J. Bosma, M. Sogaard and Y. Zhang, Maximal cuts in arbitrary dimension, JHEP 08 (2017) 051 [arXiv: 1704.04255] [INSPIRE].

[74] G.S. Joyce, On the simple cubic lattice Green function, Phil. Trans. Roy. Soc. A 273 (1973) 583.

[75] A. Sabry, Fourth order spectral functions for the electron propagator, Nucl. Phys. 33 (1962) 401.

[76] L. Adams, C. Bogner and S. Weinzierl, The two-loop sunrise graph with arbitrary masses, J. Math. Phys. 54 (2013) 052303 [arXiv: 1302.7004] [InSPIRE].

[77] R.S. Maier, On rationally parametrized modular equations, math.NT/0611041.

[78] J. Broedel, C. Duhr, F. Dulat, B. Penante and L. Tancredi, From modular forms to differential equations for Feynman integrals, in Proceedings, KMPB Conference: elliptic integrals, elliptic functions and modular forms in quantum field theory, Zeuthen, Germany, 23-26 October 2017, Springer, Cham, Switzerland (2019), pg. 107 [arXiv: 1807.00842] [INSPIRE]. 
[79] A. Pollack, Relations between derivations arising from modular forms, honors thesis, Duke University, Durham, NC, U.S.A. (2009).

[80] J. Davies, R. Gröber, A. Maier, T. Rauh and M. Steinhauser, Top quark mass dependence of the Higgs boson-gluon form factor at three loops, Phys. Rev. D 100 (2019) 034017 [arXiv: 1906.00982] [INSPIRE].

[81] M. Beneke and V.A. Smirnov, Asymptotic expansion of Feynman integrals near threshold, Nucl. Phys. B 522 (1998) 321 [hep-ph/9711391] [InSPIRE].

[82] V.A. Smirnov, Applied asymptotic expansions in momenta and masses, Springer Tracts Mod. Phys. 177 (2002) 1 [INSPIRE].

[83] M. Czakon, Automatized analytic continuation of Mellin-Barnes integrals, Comput. Phys. Commun. 175 (2006) 559 [hep-ph/0511200] [INSPIRE].

[84] A.V. Smirnov and V.A. Smirnov, On the resolution of singularities of multiple Mellin-Barnes integrals, Eur. Phys. J. C 62 (2009) 445 [arXiv:0901. 0386] [INSPIRE].

[85] M. Czakon, MB tools webpage, https://mbtools.hepforge.org/. 